A Study of the lon Hose

LA WPENCE LIWEAMCAE NATIONAL LABDAMTOPY

Instability in the DARHT-II Downstream Transport Region

J. F. McCarrick

December 15, 2004 
This document was prepared as an account of work sponsored by an agency of the United States Government. Neither the United States Government nor the University of California nor any of their employees, makes any warranty, express or implied, or assumes any legal liability or responsibility for the accuracy, completeness, or usefulness of any information, apparatus, product, or process disclosed, or represents that its use would not infringe privately owned rights. Reference herein to any specific commercial product, process, or service by trade name, trademark, manufacturer, or otherwise, does not necessarily constitute or imply its endorsement, recommendation, or favoring by the United States Government or the University of California. The views and opinions of authors expressed herein do not necessarily state or reflect those of the United States Government or the University of California, and shall not be used for advertising or product endorsement purposes.

This work was performed under the auspices of the U.S. Department of Energy by University of California, Lawrence Livermore National Laboratory under Contract W-7405-Eng-48. 


\title{
A Study of the Ion Hose Instability in the DARHT-II Downstream Transport Region
}

\author{
James F. McCarrick \\ October 4, 2004
}

\section{Introduction}

The second axis of the DARHT flash X-ray facility at Los Alamos National Laboratory ("DARHT-II") is a multiple-pulse, $18.4 \mathrm{MeV}, 2 \mathrm{kA}$ induction electron linear accelerator [1]. A train of short ( $\sim 50 \mathrm{~ns})$ pulses are converted via bremsstrahlung to X-rays, which are then used to make radiographic images at various times (nominally four) during a "hydrotest" experiment. The train of pulses is created by carving them out of a two microsecond long macropulse, using a fast switching element called a kicker [2]. The unused portion of the macropulse is absorbed in a beam dump. Thus, upstream of the kicker, two microseconds of beam are transported through a vacuum system roughly sixty meters long. These conditions involve length and, specifically, time scales which are new to the transport of high-current beams.

A concern under such conditions are the macroscopic interactions between the electron beam and positive ions created by impact ionization of the residual gas in the vacuum system. Over two microseconds, the ion density can develop to a hundredth or even a tenth of a percent of the beam density - small, to be sure, but large enough to have cumulative effects over such a long transport distance. Two such effects will be considered here: the ion hose instability, where transverse forces conspire to pull the electron beam farther and farther off axis, and background gas focusing, where radial forces (with respect to the beam) change the beam envelope during the course of the macropulse. The former effect can cause beam emittance growth (affecting the ability to focus the beam on the target) and eventually catastrophic beam loss; the latter can cause either serious degradation of the statically tuned final focus on the converter target, or a pinching of the beam on the surface of the main dump to the point where the heat flux causes damage.

The beam transport upstream of the kicker has two distinct phases. First, the beam is created and accelerated up to $18.4 \mathrm{MeV}$ over a distance of about fifty meters. Then the true downstream transport begins: the beam drifts through a matching section in preparation for the kicker, over some ten meters; the long-pulse beam then travels about four more meters from the kicker to the main dump. In the accelerator, the beam energy is obviously not constant; the transport is emittance-dominated and done through nearly continuous solenoidal focusing. In the downstream section, there are only two discrete solenoids over the entire fourteen meters and the transport is largely ballistic. Since ion hose has been studied in the accelerator [3] and since the lack of continuous focusing is considered a concern with respect to ion hose in the downstream section, the focus of this study is only from the exit of the accelerator to the main dump. A more in-depth description of the baseline (ion-free) DARHT-II downstream transport, including description of the actual transport elements and their use, will not be presented in this document; such details can be found in the documents cited in the References. 
The study of these effects will be done in stages. In the next section, the nature of the residual gas in the vacuum system will be considered, along with the various assumptions made in characterizing the creation of ions. Then the ion hose instability will be described in its simplest form. In the fourth section, additional features of ion hose will then be presented which attempt to capture some of the key behavior. Then a much more complete model using particle-in-cell (PIC) numerical techniques will be described, followed by details of the specific implementation used here. In section seven, the code will be benchmarked against results published in the literature. Section eight has the most relevant material: the actual study of the effects of ion hose and background gas focusing in the DARHT-II downstream transport region. In section nine, a simple experiment which can be tacked on to existing experiments is proposed in order to verify the modeling. Finally, the results are summarized and the very last section lists references.

\section{A Brief Examination of the Background Ion Creation}

There are two ingredients to determining the nature of the ions created in the vacuum system. One is the nature of the (neutral) residual gas in the system. The other is the ionization of that gas to create the ions. With respect to both ingredients, we will largely be presenting the work of others rather than deriving anything new from experiment or first principles.

The residual gas in the system is composed of two parts: the background gas inherent to the vacuum system (i.e., characteristic of the pumps, the vacuum protocol, the materials present, etc.), and species desorbed from surfaces during the course of the beam pulse with its attendant heating due to beam scrape. The latter will be due to more than incidental contact: in the downstream region, the main dump will directly absorb most of the beam pulse, and in the course of the kicker switching to carve out the pulse train, the beam will sweep back and forth across the "septum" that necessarily divides the long- and short-pulse beam lines.

Studies have been done to characterize both the species in the background vacuum [4] and the most likely to desorb from a heated surface [5]. In both cases, the dominant species is water. To simplify the modeling and to allow direct benchmarking with published results (Section 7), we will make the assumption that water is the only species present.

Next is the ionization model. We assume:

a) Beam impact ionization is the only mechanism - no cascading of secondary electrons and no contribution from ion-neutral collisions.

b) The net charge density (beam plus ions) is always high enough that the secondary electrons are immediately ejected to the walls, so that the neutrals are a source of positive ions only. Given the lack of any magnetic confinement in the downstream section, this should be a good assumption.

c) The depletion of neutrals due to ionization is negligible, i.e., the background neutral gas density is unchanged by ionization.

d) There is no recombination.

e) When given a background pressure in torr, the neutral density is determined from the ideal gas law at $300 \mathrm{~K}$ :

$$
n\left(m^{-3}\right)=3.3 \times 10^{22} P(\text { torr })
$$


These assumptions allow the ion density to be determined from a trivial rate equation relating the ion density to the beam density $\mathrm{n}_{\mathrm{b}}$, the neutral density $\mathrm{n}_{\mathrm{o}}$, and a cross section $\sigma$ for impact ionization:

$$
\frac{d n_{i}}{d t}=\sigma n_{o} n_{b} \beta c=\frac{\sigma n_{o} J_{b}}{q}
$$

In the case of a beam with a uniform, hard-edged profile of radius $r_{b}$ that does not vary in space or time, we can estimate the neutralization fraction $\mathrm{f}, \mathrm{n}_{\mathrm{i}} / \mathrm{n}_{\mathrm{b}}$ :

$$
f \approx \sigma n_{o} c t
$$

For the cross section, we take the value from [3], $\sigma=10^{-22} \mathrm{~m}^{-2}$. Thus at a background pressure of $10-7$ torr $\left(\mathrm{n}_{\mathrm{o}}\right.$ of $\left.3.3 \times 10^{15} \mathrm{~m}^{-3}\right)$, the neutralization fraction is

$$
f \approx 10^{-4} t(\mu s)
$$

or $2 \times 10^{-4}$ at the end of $2 \mu \mathrm{s}$, which justifies assumption (b). For a $2 \mathrm{kA}$ beam with a radius of 5 $\mathrm{mm}$ (the smallest which might occur in the downstream region), the peak ion density at the end of the pulse would be $10^{14} \mathrm{~m}^{-3}$, which justifies assumption (c).

From this, one can estimate the effect of background gas focusing on the beam. Considering only the space-charge term in the RMS envelope equation, for a $1 \mathrm{~cm}, 2 \mathrm{kA}, 18.4 \mathrm{MeV}$ beam transported 10 meters through $\mathrm{f}=10^{-4}$, we get

$$
\Delta R \approx \frac{f I z^{2}}{\gamma I_{o} R_{o}} \approx 3 m m
$$

which may be enough to influence the symmetry of the short-pulse transport through the quadrupoles in the short-pulse line, but is not threatening to long-pulse transport to the dump. The effect of higher pressures will be seen below.

\section{The Ion Hose Instability}

In this section we consider an extremely simple ion hose scenario in order to give the reader a physical picture of how the instability works. Consider a relativistic electron beam with a uniform radial current profile with hard-edge radius $r_{b}$ and charge density $\rho_{b}=-I / c \pi r_{b}{ }^{2}$. Suppose that this beam is traveling through a stationary column of ions that has the same radial profile but whose charge density is given by $\rho_{\mathrm{i}}=-\mathrm{f} Z \rho_{\mathrm{b}}$, where $\mathrm{f}$ is the neutralization fraction discussed in the previous section and $\mathrm{Z}$ is the charge state of the ions.

Let us make the incorrect but highly simplifying assumption that both the beam and the ions are completely fixed in cross section, i.e., there is no radial motion of either species with respect to 
its centroid. We will allow both the beam and the ions to shear along their transverse planes (note this is not bending since we are not allowing the normal direction of a differential slice to rotate). We consider motion in only one direction in the plane, the $\mathrm{x}$ direction. We also will ignore any axial forces that might be required for true self-consistency, so that the ions always remain in the same plane and the axial beam motion is fully specified by $\mathrm{v}_{\mathrm{z}}=\beta \mathrm{c}$, for constant $\beta$. Note that the fixed-plane assumption means that we will be ignoring the magnetic forces of the beam current on the ions, which would tend to rotate them out of their planes. Keep in mind that, in this section, all assumptions are being made to allow a simple picture, not because the assumptions are physically valid!

Now restrict our attention to what happens downstream of a fixed axial plane, which we will label as $\mathrm{z}=\mathrm{Z}_{0}$, where we will consider the beam to be "born" with a fully specified centroid position in the transverse direction. Let this position vary with time. Since we have not added any forces to our system, any given slice labeled by its injection time $t_{i}$ will retain its initial centroid position $\mathrm{x}_{0}\left(\mathrm{t}_{\mathrm{i}}\right)$ as it moves downstream. In the fixed frame of an observer in the lab, the beam will appear to wiggle as the different initial positions are convected downstream. In pictorial form, consider the centroid motion of each slice as it is born at $z_{0}$, for the case of a simple oscillation at fixed frequency $\mathrm{w}$ :

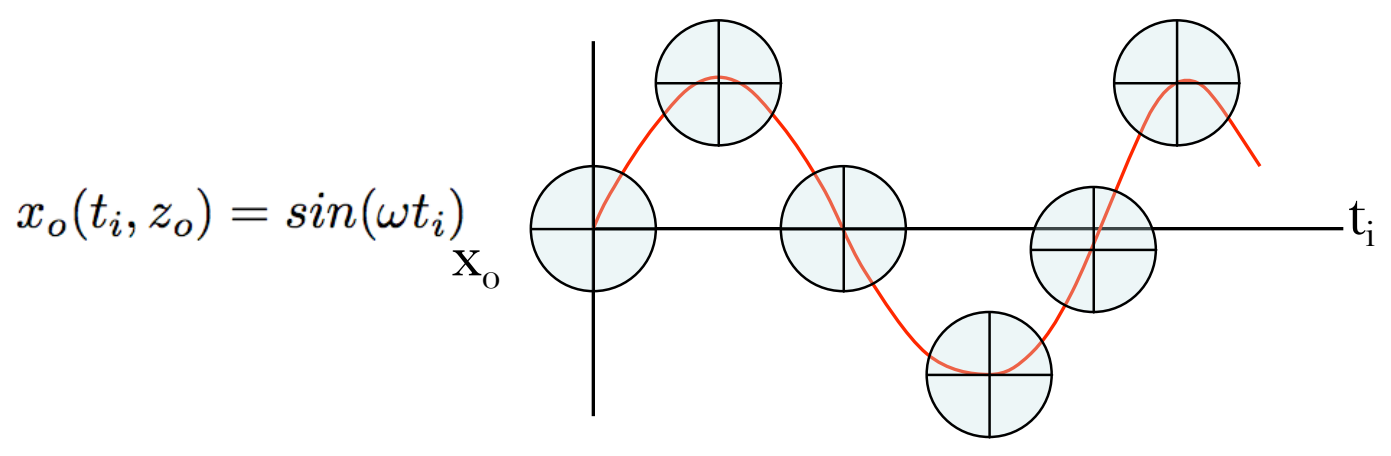

Beam cross section versus injection time at $\mathrm{z}_{0}$

At any time $t$, a beam slice at some arbitrary location $z$ must have been born at $t_{i}=t-z / \beta c$ so that the centroid location of the beam as a function of $t$ and $z$ is given by $\sin \omega(t-z / \beta c)$. A snapshot in time of our beam/ion system as function of $\mathrm{z}$ would look something like this:

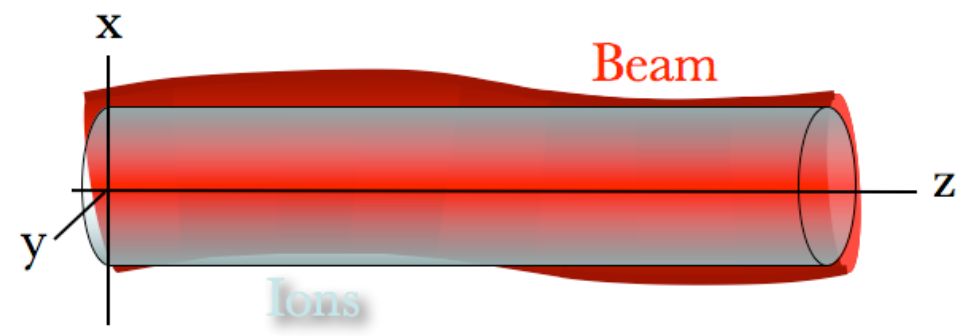

Let us now add transverse forces to the picture. We start with the ions. Under our assumption of a rigid cross section, the ions cannot exert a force on themselves. However, the beam produces a static (we assume) radial electric field which will act on the centroid of the ions. (Formally we need to average the field over the cross section of the ions but for simplicity we will neglect what 
happens at the edges and assume the average equals the value at the ion centroid $\mathrm{x}_{\mathrm{i}}$ ). As a function of displacement $\mathbf{r}$ from the center of the beam $\mathrm{x}_{\mathrm{b}}$, the electric field grows linearly from zero until the edge of the beam is reached, then decays as $1 /|\mathbf{r}|$. We will restrict ourselves to the linear regime within the beam. The direction of the field is along the displacement vector. Since we are restricting ourselves only to displacements in $\mathrm{x}, \mathbf{r}=\left(\mathrm{x}_{\mathrm{i}}-\mathrm{x}_{\mathrm{b}}\right) \mathbf{e}_{\mathrm{x}}$, the field is in the $\mathrm{x}$ direction and is given by

$$
E_{x}=\frac{-I_{b}\left(x_{i}-x_{b}\right)}{2 \pi \epsilon_{o} r_{b}^{2} c}
$$

and the equation of motion for the ion centroid is

$$
\frac{\partial^{2} x_{i}}{\partial t^{2}}=\frac{q_{i} E_{x}}{m_{i}}=\frac{Z q I_{b}\left(x_{b}-x_{i}\right)}{2 A m_{p} \pi \epsilon_{o} r_{b}^{2} c} \equiv \omega_{o}^{2}\left(x_{b}-x_{i}\right)
$$

where we use partial derivatives since $x_{i}=x_{i}(t, z) . Z$ is the ion charge state, $A$ is the atomic mass, and $\mathrm{m}_{\mathrm{p}}$ the proton mass; $\mathrm{m}_{\mathrm{i}}=\mathrm{Am}_{\mathrm{p}}$.

The resulting equation is that of a driven oscillator, which immediately sheds some light on where an instability can arise, since the oscillator has a resonance at frequency $\omega_{0}$. Without going through the exercise of solving for the behavior of a driven oscillator, we will state that the ion displacement can grow without bound if the beam centroid motion has frequency content at $\omega_{0}-$ even if the magnitude of the beam displacement is small. Of course, we are not really interested in the fate of the ions - we want to know what happens when we couple a large ion displacement back to the beam!

The above derivation holds, to a certain point, for the beam as well. The most obvious changes are using $-\mathrm{q} / \gamma \mathrm{m}_{\mathrm{e}}$ instead of $\mathrm{Zq} / \mathrm{Am}_{\mathrm{p}}$, and the scaling of the ion field strength by the neutralization fraction, f. For convenience we will still write the answer in terms of $\omega_{0}$.

$$
\frac{\partial^{2} x_{b}}{\partial t^{2}}=\frac{f A m_{p}}{Z \gamma m_{e}} \omega_{o}^{2}\left(x_{i}-x_{b}\right) \equiv \hat{f} \omega_{o}^{2}\left(x_{i}-x_{b}\right)
$$

There is a subtle problem with this result: the partial derivatives are not the same. The ion equation is taken at constant $\mathrm{z}$ while the beam equation is taken at constant $t_{i}-$ because the electron equation of motion follows individual particles and we have labeled our particles (or transverse slices of them, all particles in the slice being essentially the same) by their injection time. We can cure this mismatch by two changes of variable; we will map the ion equation from $(\mathrm{t}, \mathrm{z})$ to $\left(\mathrm{t}_{\mathrm{i}}, \mathrm{z}\right)$ and we will map the beam equation from $\left(\mathrm{t}_{\mathrm{i}}, \mathrm{t}\right)$ to $\left(\mathrm{t}_{\mathrm{i}}, \mathrm{z}\right)$. For the ions,

and the chain rule gives

$$
z=z_{\text {old }} \quad t_{i}=t-z / \beta c
$$

$$
\left.\frac{\partial}{\partial t}\right|_{z_{o l d}}=\left.\left.\frac{\partial}{\partial z}\right|_{t_{i}} \frac{\partial z}{\partial t}\right|_{z_{o l d}}+\left.\left.\frac{\partial}{\partial t_{i}}\right|_{z} \frac{\partial t_{i}}{\partial t}\right|_{z_{o l d}}=\left.\frac{\partial}{\partial t_{i}}\right|_{z}
$$

For the beam, 


$$
z=\beta c\left(t-t_{i, o l d}\right) \quad t_{i}=t_{i, o l d}
$$

and

$$
\left.\frac{\partial}{\partial t}\right|_{t_{i, o l d}}=\left.\left.\frac{\partial}{\partial z}\right|_{t_{i}} \frac{\partial z}{\partial t}\right|_{t_{i, o l d}}+\frac{\partial}{\partial t_{i}}\left|z \frac{\partial t_{i}}{\partial t}\right|_{t_{i, o l d}}=\left.c \frac{\partial}{\partial z}\right|_{t_{i}}
$$

which does not change the appearance of the equations much

$$
\begin{gathered}
\frac{\partial^{2} x_{i}}{\partial t_{i}^{2}}=\omega_{o}^{2}\left(x_{b}-x_{i}\right) \\
\frac{\partial^{2} x_{b}}{\partial z^{2}}=\hat{f} \frac{\omega_{o}^{2}}{\beta^{2} c^{2}}\left(x_{i}-x_{b}\right)
\end{gathered}
$$

but does insure that the two systems are described in a compatible set of variables. If we normalize $\mathrm{t}$ by $1 / \omega_{\mathrm{o}}$ and $\mathrm{z}$ by $\beta \mathrm{c} / \mathrm{f}^{1 / 2} \omega_{\mathrm{o}}$ we arrive at the very simple system

$$
\ddot{x}_{i}=x_{b}-x_{i}, x_{b}^{\prime \prime}=x_{i}-x_{b}
$$

where dots and primes are partial time and spatial derivatives, respectively, in the normalized coordinates (which we will still write as $\mathrm{t}$ and $\mathrm{z}$ for simplicity). Expanding in plane waves proportional to $\mathrm{e}^{\mathrm{i}(\mathrm{kz}-\omega \mathrm{t})}$ we can find a dispersion relation

$$
1=\left(1-\omega^{2}\right)\left(1-k^{2}\right)
$$

which we will solve for $\mathrm{k}$ in terms of real $\omega$ since our input to the system is essentially the behavior of the beam centroid as a function of $t_{i}$. The results are plotted below.

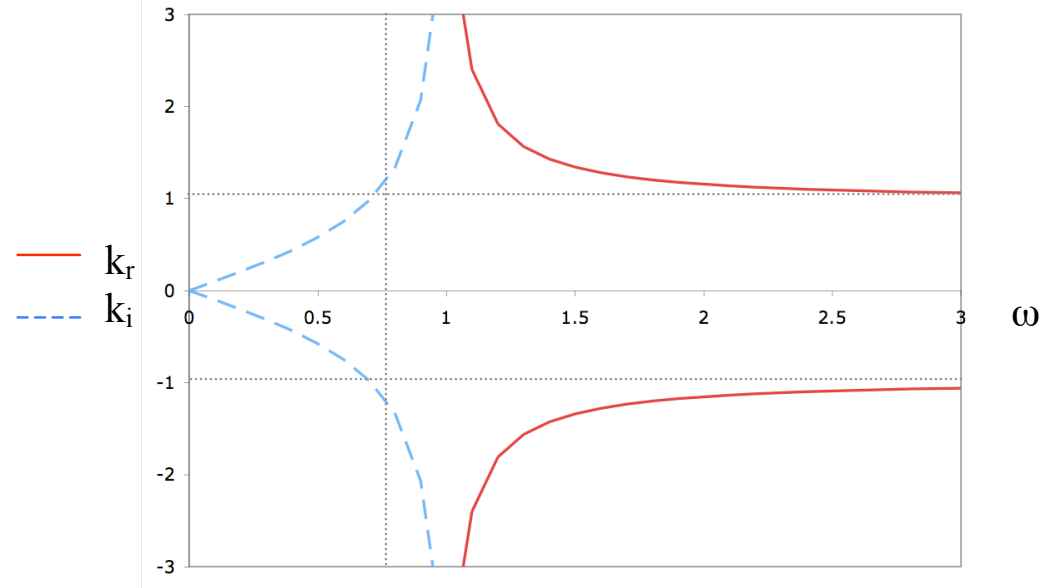

Since this is an initial value problem, turning the system on at $\mathrm{t}_{\mathrm{i}}=0$ will, in the unbounded Fourier space of the above dispersion relation, have frequency content at all frequencies even the beam oscillates like a pure sine wave at $t_{i}=0^{+}$. Thus the system is guaranteed to pick up a negative imaginary component to $\mathrm{k}$ and grow exponentially in $\mathrm{z}$. This assumption that the instability is 
indeed convective will not be proven here but the reader can find the appropriate testing procedure described in [6].

\section{Important Features of Ion Hose}

The simple picture given in section 3 is only an illustration of the basic mechanism - it does not actually have much in common with ion hose in high-current induction linacs. The good news is that most of the missing features, while complicating any attempt at pen-and-paper analysis, tend to reduce the strength of the instability. They all do so via a similar mechanism - phase mixing of many more ion components than the single fixed-area rod considered above.

How phase mixing reduces the instability can be understood by considering the previous example but with a second column of ions. Suppose (never mind why!) the motion of that column is defined by $\mathrm{x}_{\mathrm{i}, 2}=-\mathrm{x}_{\mathrm{i}, 1}$. The force on the beam is the sum of the forces from the two columns; since the force is linear in the ion position, the terms in the beam equation proportional to the ion displacement will sum to zero, leaving a simple oscillator equation for the beam as it moves with respect to an "average" ion column whose centroid is fixed at $\mathrm{x}=0$. Since the beam is described via initial conditions in time, not space, there is no way to drive the oscillator in a resonant fashion and the system is stable.

Here are some sources of phase mixing in real ion hose. The reader will undoubtedly be able to think of more:

- Ions are created across the entire cross section of the beam. Thus, each particle starts with zero velocity but at a different position in the potential well - the average particle position is always the beam centroid (but only at the time of creation).

- Ions are created continuously in time. Early in the pulse there are so few ions that their influence on the beam, regardless of their offset, is negligible; at any time, a population of ions is being created with zero average offset from the beam.

- The force between the beam and ions is not linear. There is no single frequency that characterizes the ion motion and the transverse distribution of both the beam and ions is not uniform.

- The ion population does not form a shape with rigid cross section - far from it! A set of ions born across the area of a beam with circular cross section will exhibit radial motion on a fast time scale.

- The ion population does not consist of a single species. Even in linear forces, each species will have a different characteristic frequency because of the dependence on mass. In fact, this is the inspiration for the spread-mass model [7], which approximates the nonlinear motion of a single species with a carefully weighted range of frequencies (masses), meant to capture the spectrum of the anharmonic motion, but in a linear system.

Other effects are not linked to phase in the transverse plane, but in the axial direction. If the average ion position in $\mathrm{z}$ is reduced, the effect on the beam can be reduced (but not as a general rule, since $\mathrm{z}$ variation in the ion forcing terms can couple resonantly to the beam at arbitrarily small amplitude). Some effects along these lines are: 
- The beam and ion motion cannot in general be decoupled into $\mathrm{x}$ and $\mathrm{y}$ planes. The presence of an axial magnetic field will cause a radial force to lead to orbital rather than purely radial motion. - The beam will not in general have a fixed radius as it propagates axially. Even in the simple linear picture, the frequency of the ion motion will vary with $\mathrm{z}$ and so the average ion position will not have fixed phase.

Simple illustrations of some of these phenomena are as follows.
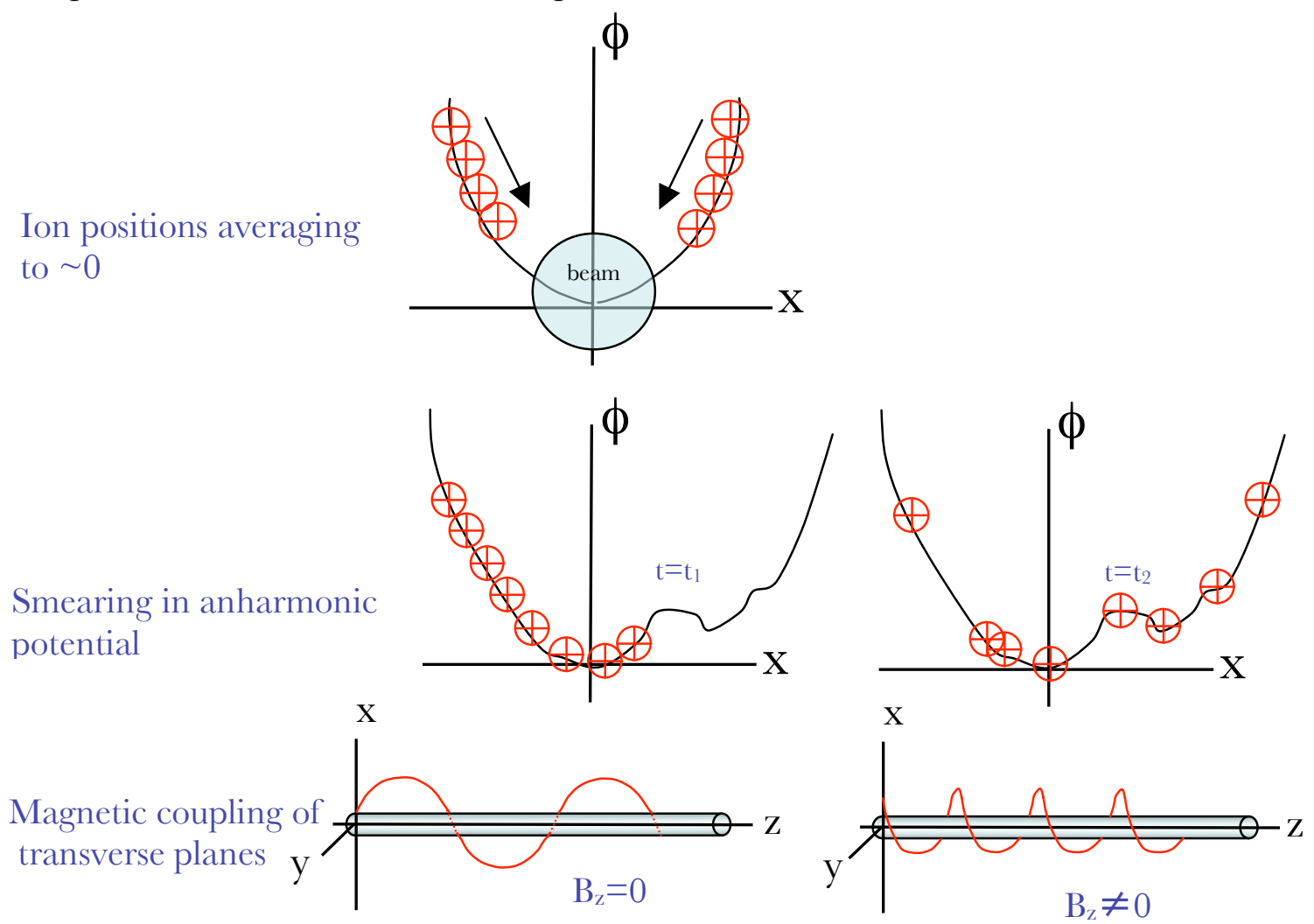

Many of the above effects have been studied on an individual basis, both in references already cited and (particularly regarding envelope variation) in [8]. However, to study them all requires resolution of the ion phase space that makes analytic study extremely difficult. Without dwelling on additional algebra, then, we will turn to numerical modeling.

\section{PIC Modeling of Ion Hose and Background Gas Focusing}

Particle-in-cell (PIC) simulation of ion hose allows a much fuller treatment than any tractable analytic approach. To perform the studies given in the remainder of this document, the ADCGlib beam physics library, an object-oriented toolkit written in $\mathrm{C}++$ and developed at LLNL, was expanded to include some specialized facilities for large-scale ion hose simulations.

The main advantage of PIC is that it allows one to follow the evolution of the detailed phase space of both the beam and the ions, with reasonable resolution. Note that the details which follow are those used in these particular ion hose simulations and do not represent the way in 
which all beam simulations are done with ADCGlib - the library has a variety of models available.

The beam is divided into transverse slices, each slice representing a particular time $t_{\mathrm{i}}$ at which particles enter the system. The slice is composed of macroparticles, each representing a large number of electrons, sampled from a given initial phase space $f\left(x, y, p_{x}, p_{y} ; p_{z}, z_{0}, t_{i}\right)$. The phase space $\mathrm{f}$ is normalized so that each slice carries a current I. The vector components of $\mathbf{p}$ are the normalized relativistic momenta, $\gamma \mathbf{v} / \mathrm{c}$.

Particles are "pushed" via a fourth-order Runge-Kutta step according to the Lorenz force, written in terms of propagation in the axial direction:

$$
\begin{gathered}
\frac{d \vec{x}}{d z}=\frac{\vec{p}}{p_{z}} \\
\frac{d \vec{p}}{d z}=\frac{-q \gamma}{p_{z} m_{e} c^{2}}\left(\vec{E}+\frac{c}{\gamma} \vec{p} \times \vec{B}\right)
\end{gathered}
$$

The fields are assumed quasi-static and therefore constant over the (very short) time interval of a particle step $\sim \Delta \mathrm{z} / \mathrm{c}$. They are also assumed to be paraxial; that is, within any transverse slice of the problem it is assumed that the charge and current densities, determined by the PIC statistics of the beam and ions at that slice, do not vary in the axial direction. The applicable field equations are

$$
\begin{gathered}
\vec{E}=E_{e x t} \hat{e}_{z}-\vec{\nabla} \phi \\
\vec{B}=\vec{B}_{e x t}+\vec{\nabla} \times A \hat{e}_{z} \\
\nabla_{\perp}^{2} \phi=-\frac{\rho_{b}+\rho_{i}}{\epsilon_{o}} \\
\nabla_{\perp}^{2} A=-\mu_{o} J_{z, b}
\end{gathered}
$$

where the Laplacian operates on the transverse coordinates only. The subscript "ext" indicates that these components are fully specified external fields. Note that the ions do not contribute to the axial current.

The pipe is taken to be a perfect conductor with a circular cross section. This sets the boundary conditions that both the scalar and vector potential must go to zero on this surface. Solution of the field equations is done by breaking the sources into Fourier modes in $\theta$ via FFT and solving the resulting set of uncoupled radial equations.

The ions are sampled and pushed via PIC similarly to the electrons, but with additional assumptions. There is no axial velocity. The motion is assumed non-relativistic. For the downstream transport study but not the benchmarking runs, the ions are also assumed to be unmagnetized, for reasons to be explained later (even in the magnetized case, only $\mathrm{B}_{\mathrm{z}}$ is used). The ions are born with zero initial velocity, so the phase space from which they are sampled is just the density of ions created within a time step $\Delta t$ in accordance with the model outlined in section 2: 


$$
n_{i, b o r n}(r, \theta, z, t)=\Delta t \frac{\sigma n_{o}(z) J_{b}(r, \theta, z, t)}{q}
$$

The sampling process is carried out in two steps. First, a low-resolution sweep of the bounding rectangle of the beam is carried out in order to estimate the beam area, which is required to convert the local ion density to an actual number of macroparticles. Then the desired number of samples (new ions per time step) is done within the area of the beam. Successful samples are weighted by the beam current density; samples at locations of zero density are rejected.

The physical system (as already implied by the paraxial field assumption) is itself divided into slices of finite thickness (the beam slices are essentially slices of finite time) in the axial direction. To avoid confusion with the beam slices, the axial slices will hereafter be referred to as "chunks". Each such chunk has its own population of ions that evolves independently of the other chunks. Consider the collection of beam slices to be a deck of cards. Each card is fed, one by one, into a given chunk of pipe. The passage of one card represents the passage of a fixed amount of time at that axial location. The deck of cards is collected at the output of that axial chunk, then fed into the next, until the beam has been transported through the entire domain. In this picture, only one axial chunk of ions needs to evolved at any given stage of the process.

Transport through chunk at $\mathrm{z}$ Transport through chunk at $\mathrm{z}+\Delta \mathrm{z}$
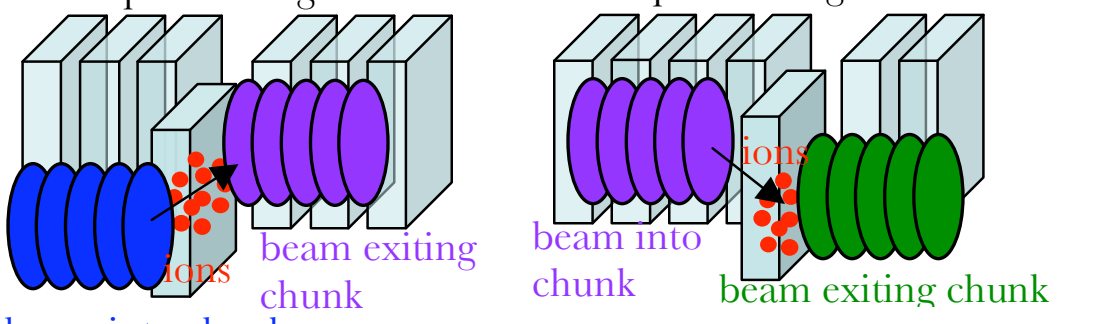

beam into chunk

One additional bookkeeping process is required in order to maintain tractability of the problem, even assuming considerable computational resources. To give a feel for the scope, suppose the number of new ions created at each time step is fairly modest, say 40 . With time resolution of 0.1 $\mathrm{ns}$, the population of ions at each axial chunk is $8 \times 10^{5}$ by the end of the run. Suppose that, on average, half that number is pushed at each time step. Then a $50 \mathrm{~m}$ simulation with $3 \mathrm{~cm}$ resolution (as done for the benchmark runs, see section 7 ) will involve $\sim 10^{13}$ macroparticle steps before all is said and done - the work in pushing the beam is almost negligible. Given that each macroparticle step involves several degrees of freedom and various field evaluations, this is a very large number of operations to complete within any reasonable amount of time. Thus, to reduce the scope of the problem almost an order of magnitude, the ion population per chunk is capped at about $10^{5}$. Whenever the population reaches $1.1 \times 10^{5}, 10^{4}$ macroparticles are chosen at random and discarded; the sum of their charge is evenly distributed among the remainder. Since the down select is done randomly, no attempt is made to force conservation of any quantity other than charge. 


\section{Numerical Details}

The following parameters are used in all simulations that follow:

$\begin{array}{ll}\text { Time resolution } & 0.1 \mathrm{~ns} \\ \text { Axial resolution }=\Delta \mathrm{t} / \mathrm{c} & 3 \mathrm{~cm} \\ \text { Number of radial grid points } & 150 \\ \text { Number of azimuthal modes } & 128 \\ \text { Number of macroparticles per beam slice } & 3000 \\ \text { Number of ion macroparticles created per time step, per slice } & 40 \\ \text { Beam current } & 2 \mathrm{kA} \\ \text { Ion charge (in units of q) } & 1 \\ \text { Ion atomic mass } & 18\end{array}$

Note that the axial resolution is tied to the temporal resolution. This is not a Courant condition in the usual sense (the quasi-static field calculation is stable regardless of step size) but linked to the fact that the time resolution is set by how fast the ion phase space is evolving. Since time is a fixed parameter for the beam Runge-Kutta push (which, recall, is done in $\mathrm{z}$, not $\mathrm{t}$ ), the largest spatial step for the beam corresponds to the longest amount of time over which the fields are considered unchanged - which is the time step for the ions. A more sophisticated push technique would allow these quantities to be uncoupled, especially since the spatial variation for the parameters of interest is very long wavelength; but the present technique was ready to use without any additional bookkeeping or code development.

The radial resolution can be determined once the pipe size is known. For the benchmarking studies, a pipe radius of $6 \mathrm{~cm}$ was used, giving a resolution of $0.4 \mathrm{~mm}$; for all other runs, an $8 \mathrm{~cm}$ radius was used, for a resolution of $0.53 \mathrm{~mm}$.

The number of azimuthal modes has been criticized by some as "excessive" and that only monopole and dipole terms are significant. Given that the simulations are meant to capture emittance growth in beams that can move off axis by their own diameter, this is incorrect. So long as the center of the polar system is the pipe origin and not the beam centroid - which must be the case since the pipe sets the boundary conditions - high order is required to capture the field structure at the location of the beam. Another way of seeing this is that for large beam displacements, the $\mathrm{X}$ and $\mathrm{Y}$ directions in a Cartesian frame would have to have similar resolutions; the same remains true in a Fourier decomposition. In this case, the advantage to the Fourier decomposition is simply the computational efficiency of the FFT given the boundary conditions, not because it is a more efficient representation of the eigenmodes.

The simulation algorithm was made parallel by the use of the Message Passing Interface (MPI). An assembly line technique was used which allowed very good load balancing and a minimum of inter-process communication. Consider a simulation of axial length $\mathrm{L}$ which is broken into $\mathrm{N}_{\text {tot }}=\mathrm{L} / \Delta \mathrm{z}$ axial chunks. If $\mathrm{N}$ processors are available, each is responsible for $\mathrm{N}_{\mathrm{c}}=\mathrm{N}_{\mathrm{tot}} / \mathrm{N}$ contiguous chunks. (Since $\mathrm{N}_{\mathrm{tot}} / \mathrm{N}$ is not in general an integer but $\mathrm{N}_{\mathrm{c}}$ must be, the total simulation ends up covering a length slightly shorter than $\mathrm{L}$.) The beam is represented by $\mathrm{N}_{\mathrm{s}}$ slices in time. 
The initial state of these slices is determined by the incoming phase space and the initial perturbation put on the beam centroid. One beam slice at a time is transported through all $\mathrm{N}_{\mathrm{c}}$ chunks of a given processor, then handed off to the next processor. The first processor creates the incoming beam slices as it needs them; later processors receive these slices one at a time; thus, storage is dominated by the field solutions and the ions. For the resolutions quoted above, a $50 \mathrm{~m}$ simulation spread over 32 processors requires on the order of $300 \mathrm{MB}$ of RAM per processor, a modest requirement. At any given time, the relevant quantities of interest are output to disk rather than stored in memory.

The main algorithm that drives the various ADCGlib components is listed in the Appendix. The ADCGlib objects themselves are not given.

\section{Benchmarking the Code}

Since the ion-related objects and FFT solver are new to ADCGlib, it is imperative to benchmark the code against a known quantity in order to believe the results of such a large-scale simulation. This was done by comparing ADCGlib simulations to results published in the literature using the commercial code LSP [3]. The cases considered in the reference are meant to represent not the downstream transport but the accelerator itself, and are hence a much longer distance, some 50 meters. The particular runs chosen for comparison are the constant-energy cases with uniform background pressure. The beam energy is $12.5 \mathrm{MeV}$, representing the average value in the accelerator, and has a constant RMS radius of $5 \mathrm{~mm}$ and an emittance of $1000 \mathrm{~mm}-\mathrm{mrad}$, normalized Lapostolle ( $2 \times 10^{-5} \mathrm{~m}$-rad un-normalized Lee-Cooper). The corresponding magnetic field for matched transport, found by considering the RMS envelope equation, is

$$
B=\frac{2 \gamma m_{e} c}{q R} \sqrt{\frac{\epsilon^{2}}{R^{2}}+\frac{I}{\gamma^{3} I_{o}}}
$$

which gives a value of $0.083 \mathrm{~T}$. In this expression $\mathrm{I}_{\mathrm{o}}$ is the Alfven current for electrons, $\sim 17 \mathrm{kA}$.

The initial perturbation on the beam is a fixed-frequency oscillation in one plane with an amplitude of $1 \%$ of the radius, $50 \mu \mathrm{m}$. The chosen frequency is $21.6 \mathrm{MHz}$; the full explanation is given in the reference, but the basic explanation is that this corresponds to maximum growth according to the spread-mass model.

The input phase space for the ADCGlib runs is KV but the input phase space for the LSP runs is Gaussian. This is, strictly speaking, a mistake in the benchmarking process and it has not been addressed due to time and funding limitations. However, some comparisons done for the downstream transport region, discussed in the next section, indicate that the physics is not terribly sensitive to the beam distribution - a fortunate turn of events, since we have no way of knowing what the DARHT-II phase space will actually look like. (As often as people scoff at the unphysical nature of a KV beam, the author has never seen a truly Gaussian one, either.)

The resolution of the LSP simulations in the transverse direction is lower by a factor of about three, $1.25 \mathrm{~mm}$ versus 0.4 . The axial resolution is much lower, $30 \mathrm{~cm}$ versus 3 . The resolution of 
the ion "birth" phase space is very much lower, 1 macroparticle per time step rather than 40. These numbers are not in the reference but come from [9].

The runs are done at three background pressures, $1 \times 10^{-7}, 3 \times 10^{-7}$ and $6 \times 10^{-7}$ torr. The ADCGlib run times are about two days on 32 CPUs, per case. The output quantities available for comparison are the centroid of the beam and the ion column, plotted as a function of time at the fixed location of the accelerator exit. The figures are on the following pages.

Overall, the agreement between the codes is acceptable but not spectacular. The nonlinear saturation amplitudes agree well but the linear growth rate tends to be slower in the ADCGlib runs, especially at the lower pressures. The ADCGlib curves appear stretched out relative to their LSP counterparts. It is unclear at this time what could cause a difference that is noticeable but not enormous.

- The lower spatial resolution of the LSP runs should still be adequate to capture the centroid motion (although perhaps not emittance growth, but that is not compared here).

- The lower ion phase space resolution in LSP may be a factor; the use of the KV phase space with ADCGlib may also be a factor.

- For these runs, the $\mathbf{v} \times \mathrm{B}_{\mathrm{z}}$ term was retained in the ADCGlib equation of motion for the ions, whereas the LSP ions are unmagnetized, but the latter is a good assumption and is not likely to have produced a large difference.

- The details entering the nonlinear regime may be dependent on the phase of the initial perturbation.

- The $6 \mathrm{~cm}$ pipe size used with ADCGlib is much smaller than the pipe size in the DARHT-II accelerator $(\sim 12 \mathrm{~cm})$, and at low values of the neutralization fraction, the image forces that an offset beam experiences from the pipe wall at this size are in fact comparable to the ion hose contribution (this is not true for the entire pulse).

However, since these runs are the most time-consuming of any required by this entire study and since additional time and funding are not available, the agreement is considered "good enough" and we shall move on to the downstream transport region. 

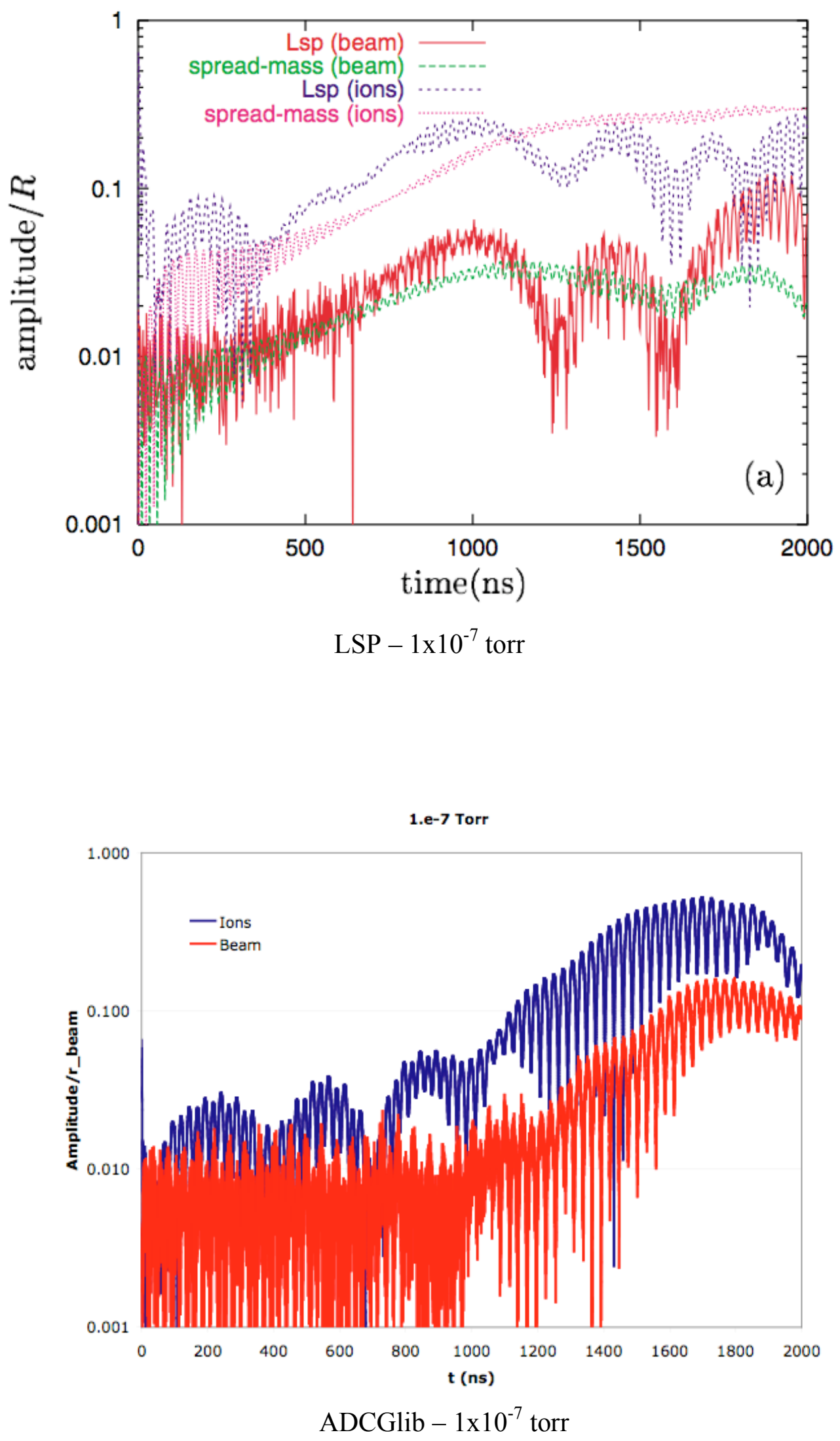


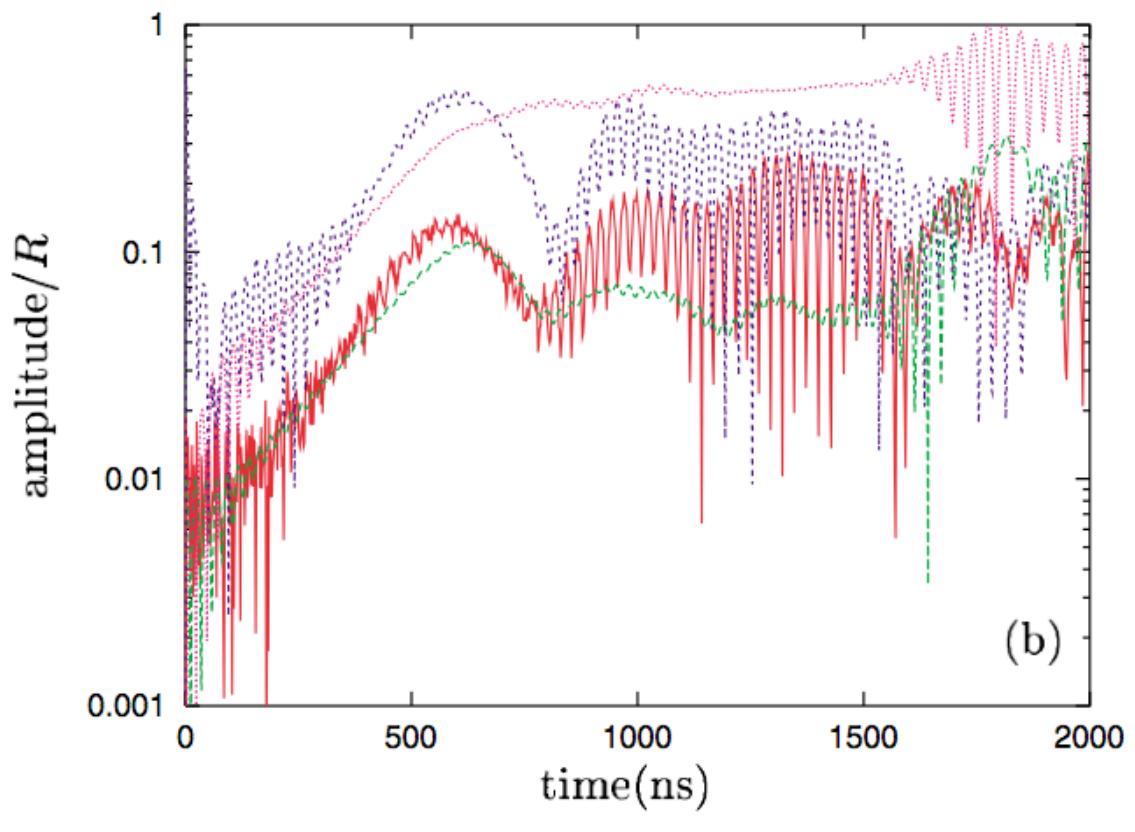

$$
\text { LSP }-3 \times 10^{-7} \text { torr }
$$

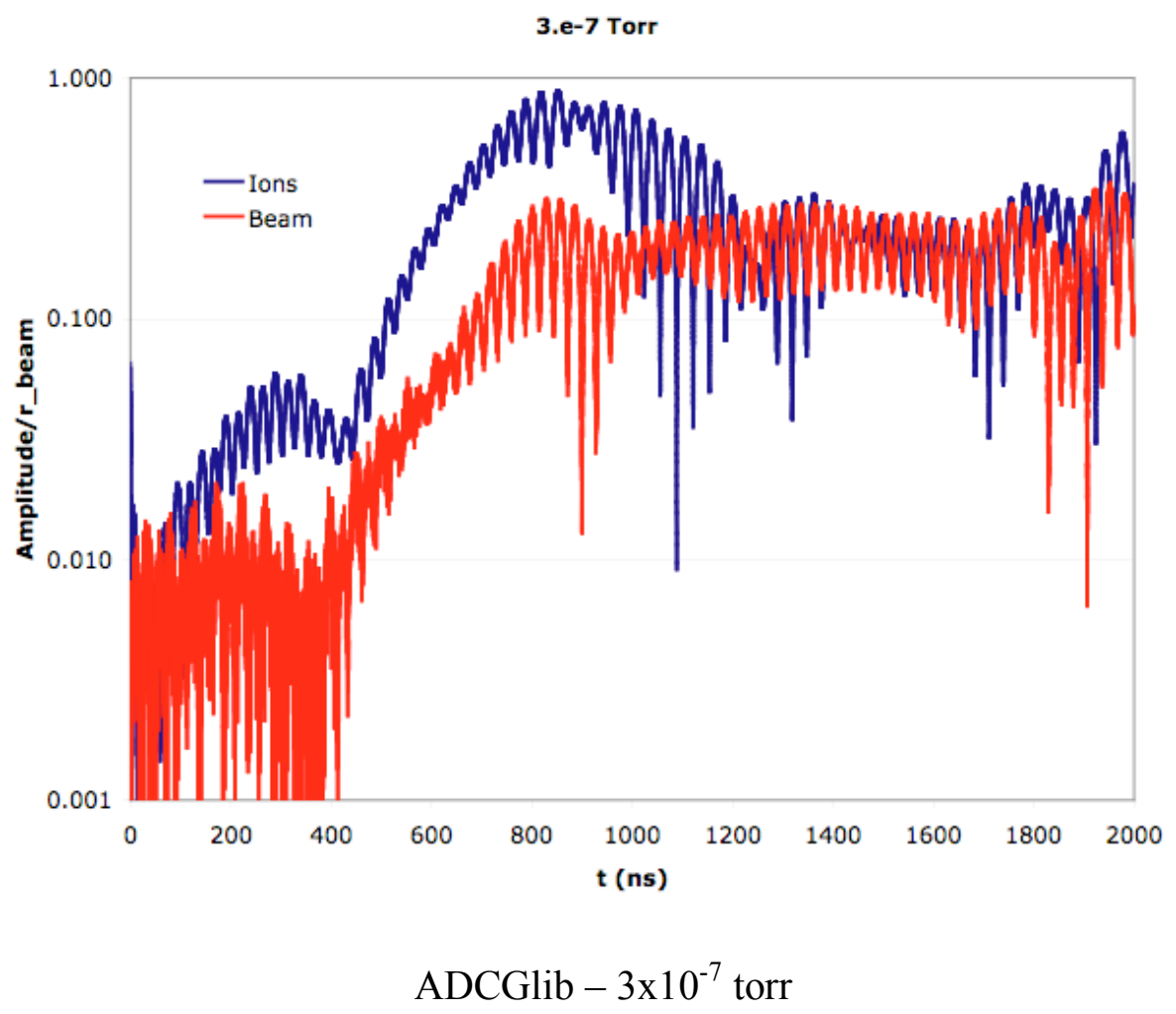




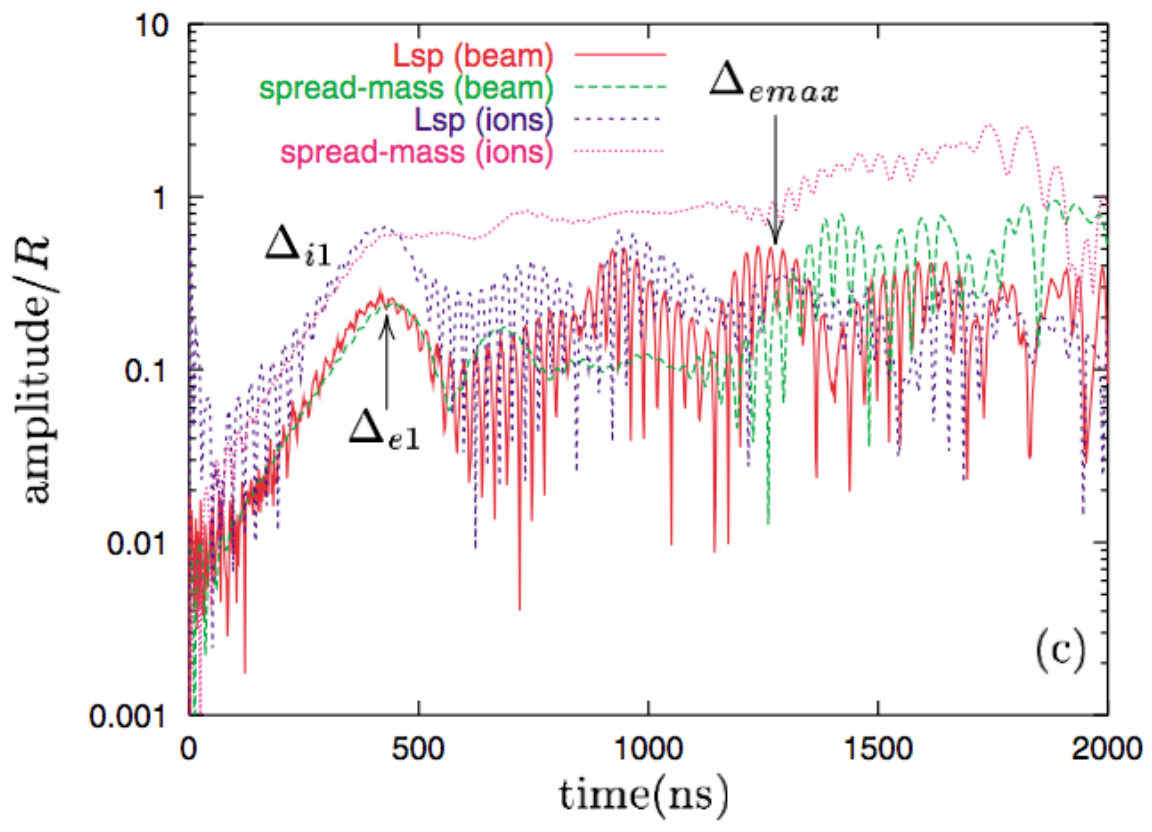

$$
\text { LSP }-6 \times 10^{-7} \text { torr }
$$

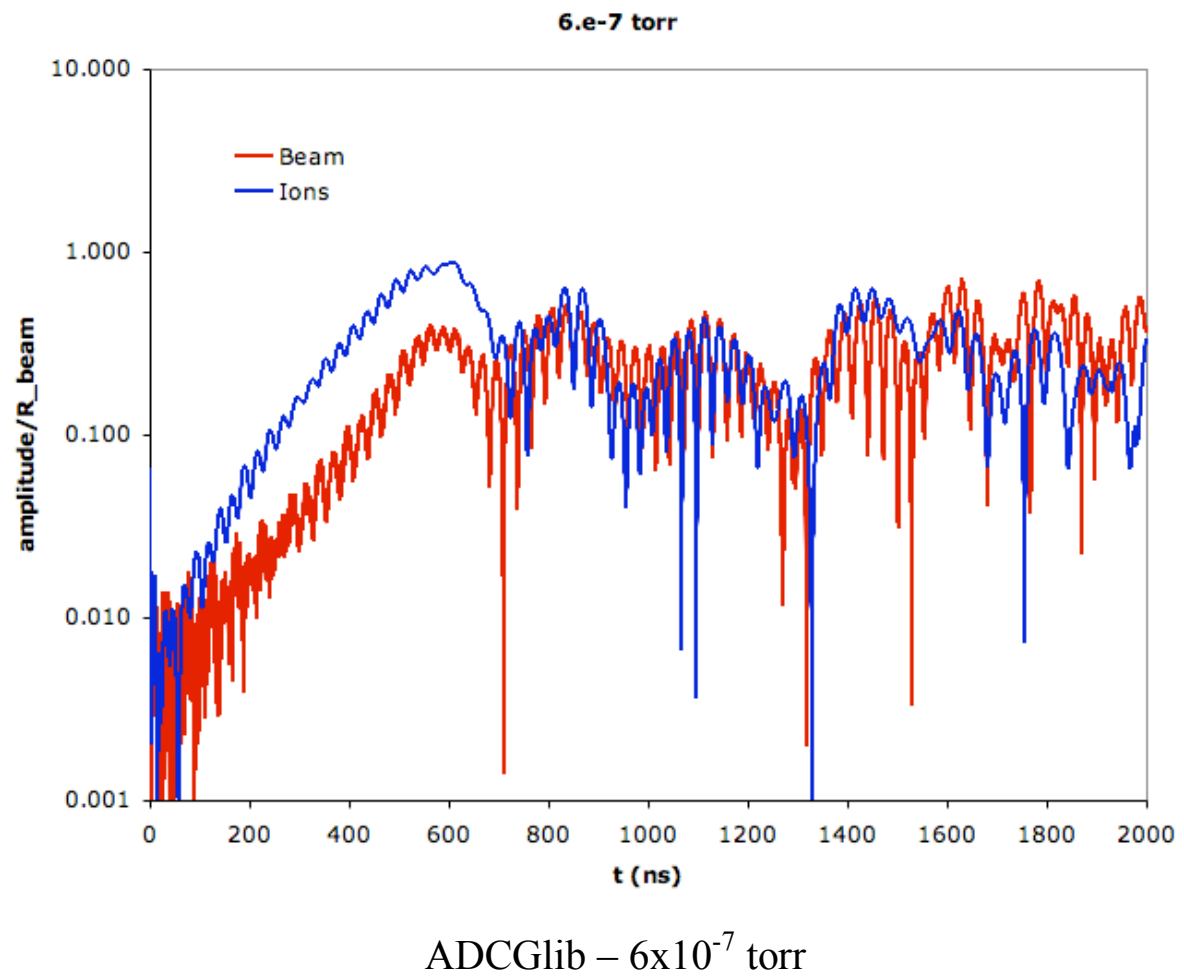




\section{Downstream Transport Study}

In this section, we deal with the main concern of this report: transport in the downstream region of DARHT-II, in the long-pulse line to the main dump. We consider four sets of cases. In the first, the beam is transported through the predicted baseline vacuum conditions. In the second, transport is through localized perturbations of that baseline, simulating a local pressure rise due to outgassing in the septum and dump area. In the third, to get a general feel for the overall pressure requirements in case the predicted profile is not accurate, the beam is transported through uniform profiles of various pressures. In the fourth, a couple of cases from the first set are reconsidered but with a Gaussian beam phase space; the first three are done with a KV beam.

These simulations differ from the benchmarking runs in a few ways. First, the pipe size has been increased to the correct value of $8 \mathrm{~cm}$. Second, the initial beam perturbation is not at a fixed frequency but is random with a flatband power spectrum from 0 to $100 \mathrm{MHz}$; the RMS value of the transverse displacement is one percent of the initial radius, and the phase in the transverse plane varies randomly. Details of how this is generated can be read directly from the source code in the Appendix. The third difference is that the ion equation of motion does not have the magnetic field term. This is a good approximation but was done for less honorable reasons: since the magnetic field is localized to small sections of the total, and since computation time is dominated by pushing ions, MPI load balancing would be broken because some processes would need to evaluated field quantities that others would not. The fourth difference is the beam energy, which outside the accelerator is obviously at its maximum value of $18.4 \mathrm{MeV}$. Finally, the emittance is at the specification for the downstream transport, $1500 \mathrm{~mm}$-mrad normalized Lapostolle.

\subsection{The Baseline Case}

The baseline pressure profile, assuming no gas desorption due to localized beam heating during the course of the pulse, is taken from [10]; it is plotted below. The average pressure is about $8 \times 10^{-8}$ torr. A detailed transport layout will not be given here, for the reasons stated in the introduction, but the following conditions are used: the beam size entering the calculation is 3.54 $\mathrm{mm}$ ( $5 \mathrm{~mm}$ edge); the $\mathrm{S} 0$ solenoid is set to $0.187 \mathrm{~T}$; the $\mathrm{S} 3$ solenoid is set to $0.177 \mathrm{~T}$. Since ADCGlib is constrained to round pipe cross sections and since the beam moves far off axis following transport through the kicker, the quad septum, and the dipole ahead of the main dump, there are no quadrupole or dipole elements included in this simulation. Thus the beam stays round going into the dump even though this would not be the case in reality. This is not considered germane to the question of ion hose.

The baseline beam envelope, in the absence of any ions, is also given below along with the corresponding calculation in [11]; they agree extremely well. Note that the Transport results from [11] appear shorter in length but this is because they follow the true bend of the beam centroid for a path length of about $14 \mathrm{~m}$ but are plotted versus the projected $\mathrm{Z}$ coordinate. 

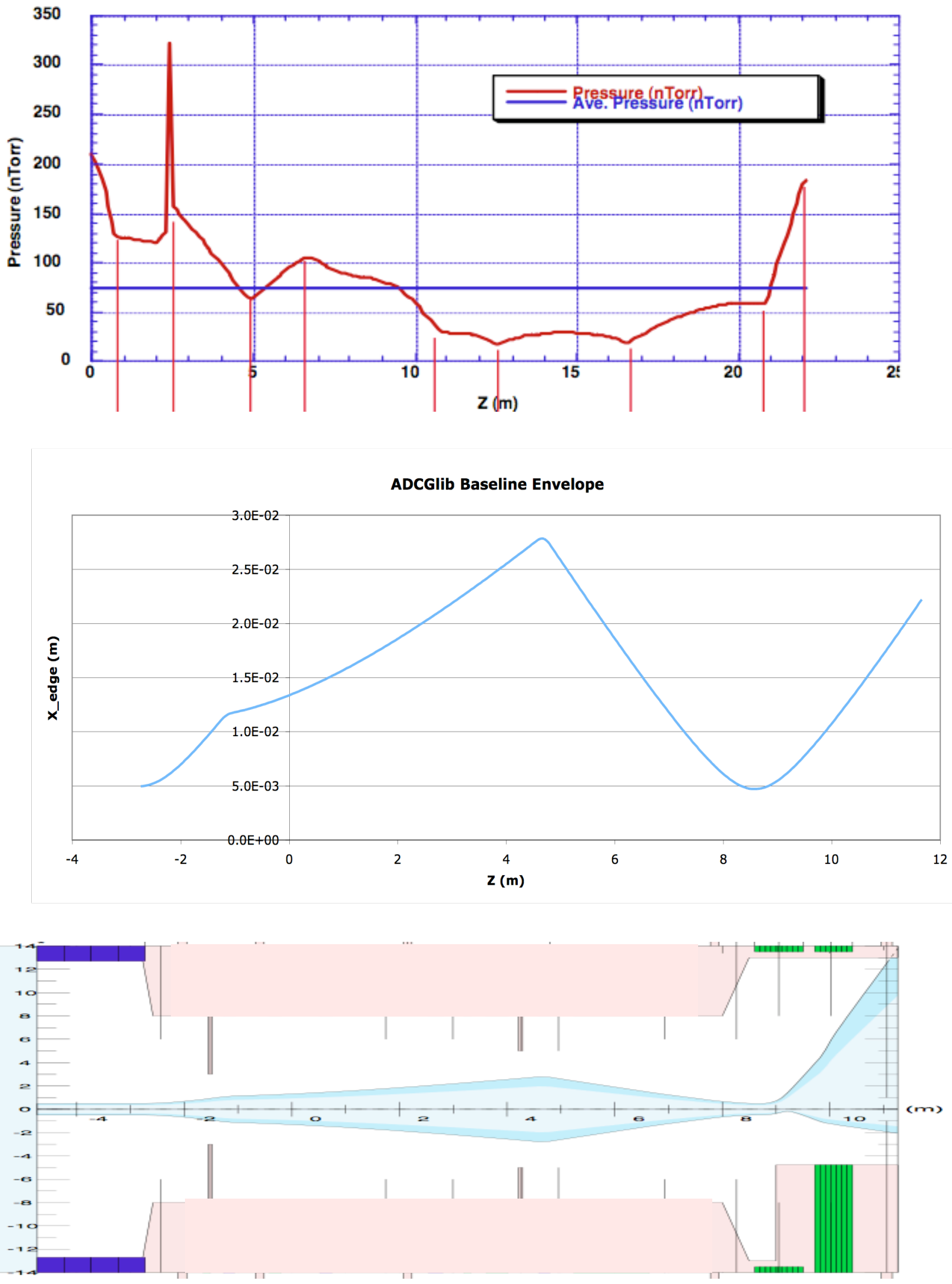
The quantities of most interest in these simulations are the centroid motion, the beam emittance, and any change in the beam envelope due to radial gas focusing forces. The emittance and envelope are plotted below versus $\mathrm{z}$, at $\mathrm{t}=0$ and $\mathrm{t}=2 \mathrm{~ms}$. The beam centroid location is plotted over the course of the pulse at $\mathrm{z}=0$ and $\mathrm{z}=14 \mathrm{~m}$.
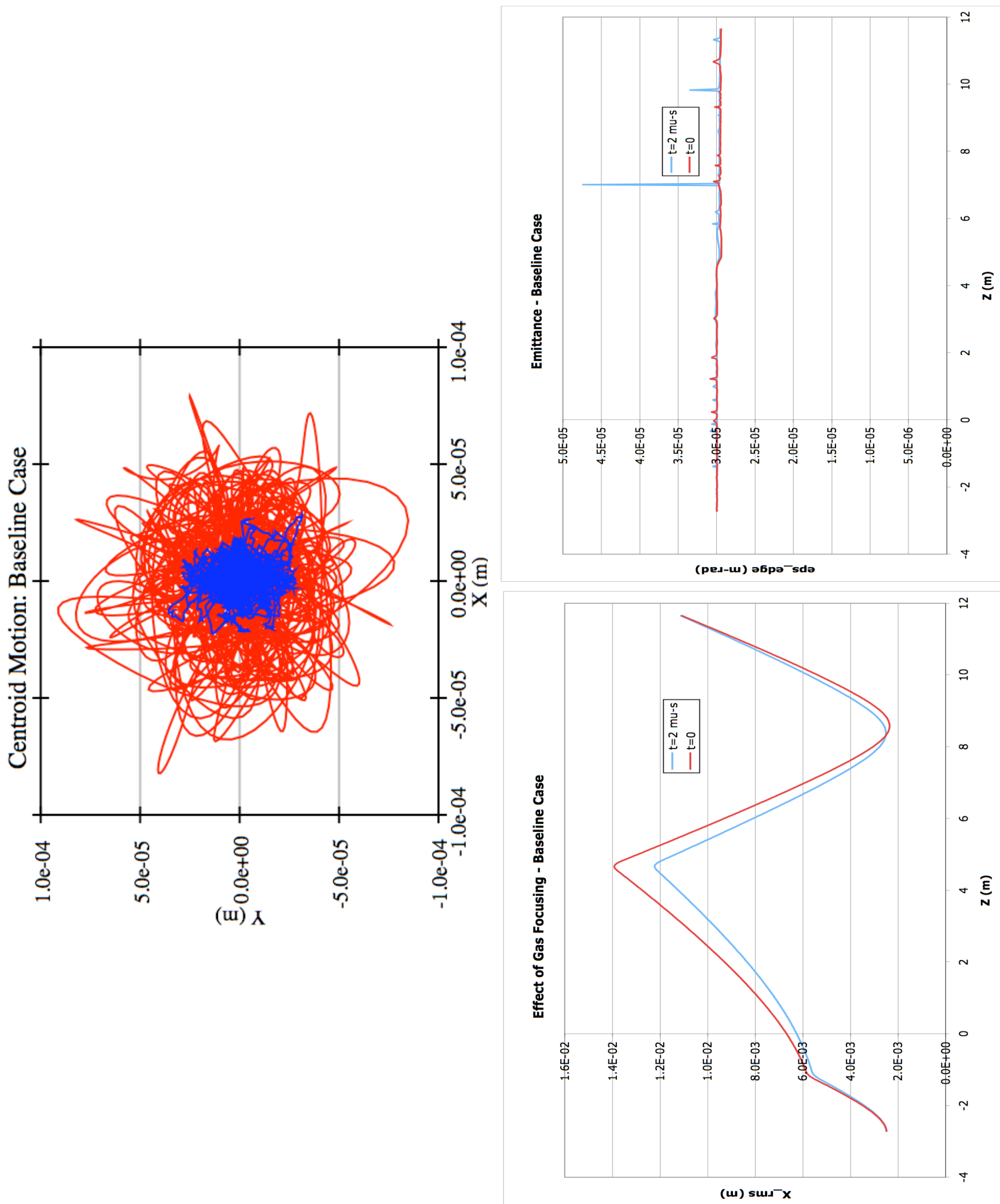
Thus, from these results, neither ion hose nor gas focusing is a major concern in the baseline configuration. The change in the beam envelope is not negligible with respect to transport through the short pulse line, but it can be dealt with in that section and does not pose a threat to the main dump from excessive heat loading by a pinched beam. The emittance is completely unaffected (the spikes in the curve are numerical artifacts from the de-spinning algorithm used in ADCGlib to account for a beam with correlated $\mathrm{X}$ and $\mathrm{Y}$ directions in a magnetic field). The centroid motion actually appears to damp - although this is not quite the case. Let us consider another way of examining the centroid behavior: treating the centroid position as a complex signal $\mathrm{X}(\mathrm{t})+\mathrm{iY}(\mathrm{t})$ at a given location and looking at its power spectrum. The input spectrum of the flatband signal described at the beginning of this section looks as follows:

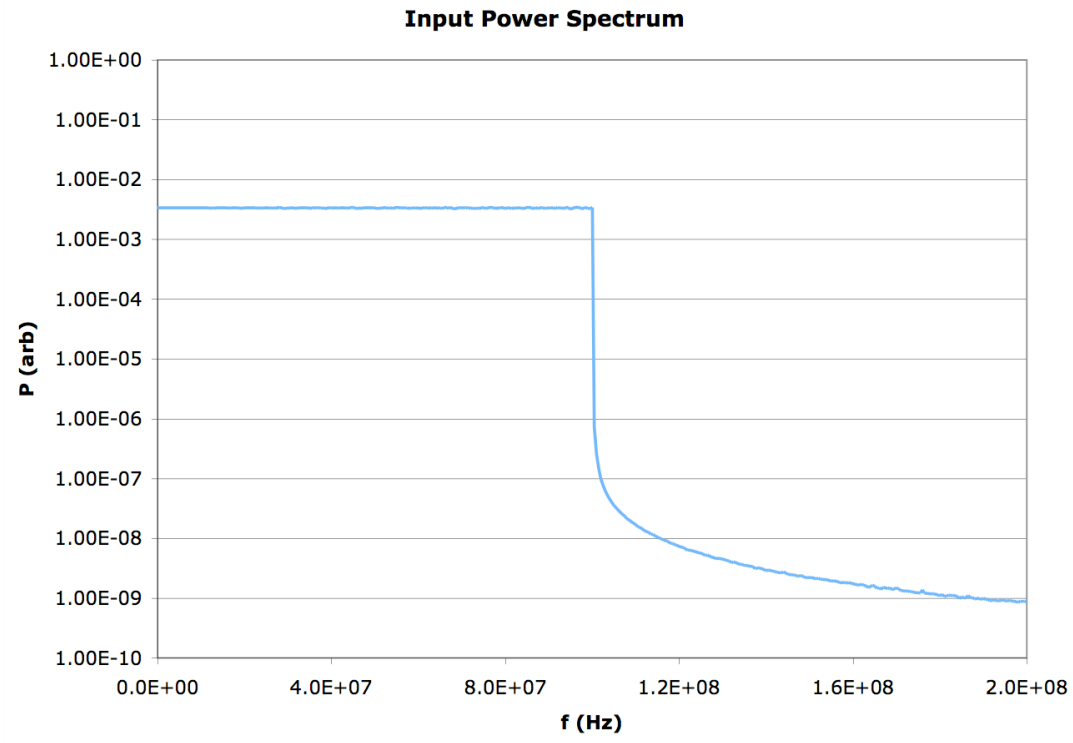

The spectrum at the location of the dump can be examined the same way; we can see the relative growth at a given frequency by looking at the normalized spectrum $\mathrm{P} / \mathrm{P}(\mathrm{z}=0)$. The result for the baseline case indeed shows damping at many frequencies but growth in a region in the vicinity of $25 \mathrm{MHz}$ :

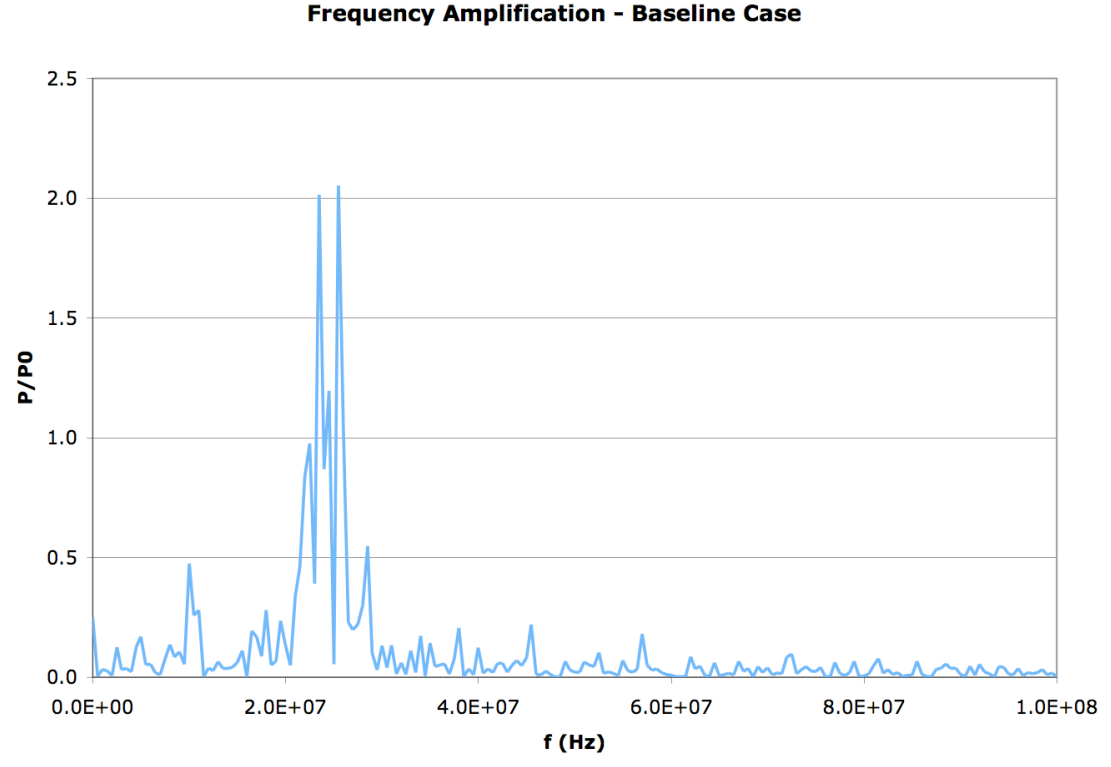




\subsection{Localized Pressure "Transients"}

Let us now consider the effects of gas desorption from the septum walls and the main dump as the beam deposits energy on these surfaces during the course of the long pulse. We will do this by incorporating a localized pressure rise, relative to the baseline, at the end of the transport line, that is present at $\mathrm{t}=0$ (so it is not really a transient). To bound it in space, we will assume that it extends no farther upstream than the first pumping station after the kicker. The values chosen for the simulations are $5 \times 10^{-7}, 1 \times 10^{-6}$, and $5 \times 10^{-6}$ torr, to insure that the threshold for unacceptable behavior is found. These modified pressure profiles, along with the baseline, are shown on a log scale in the following plot.

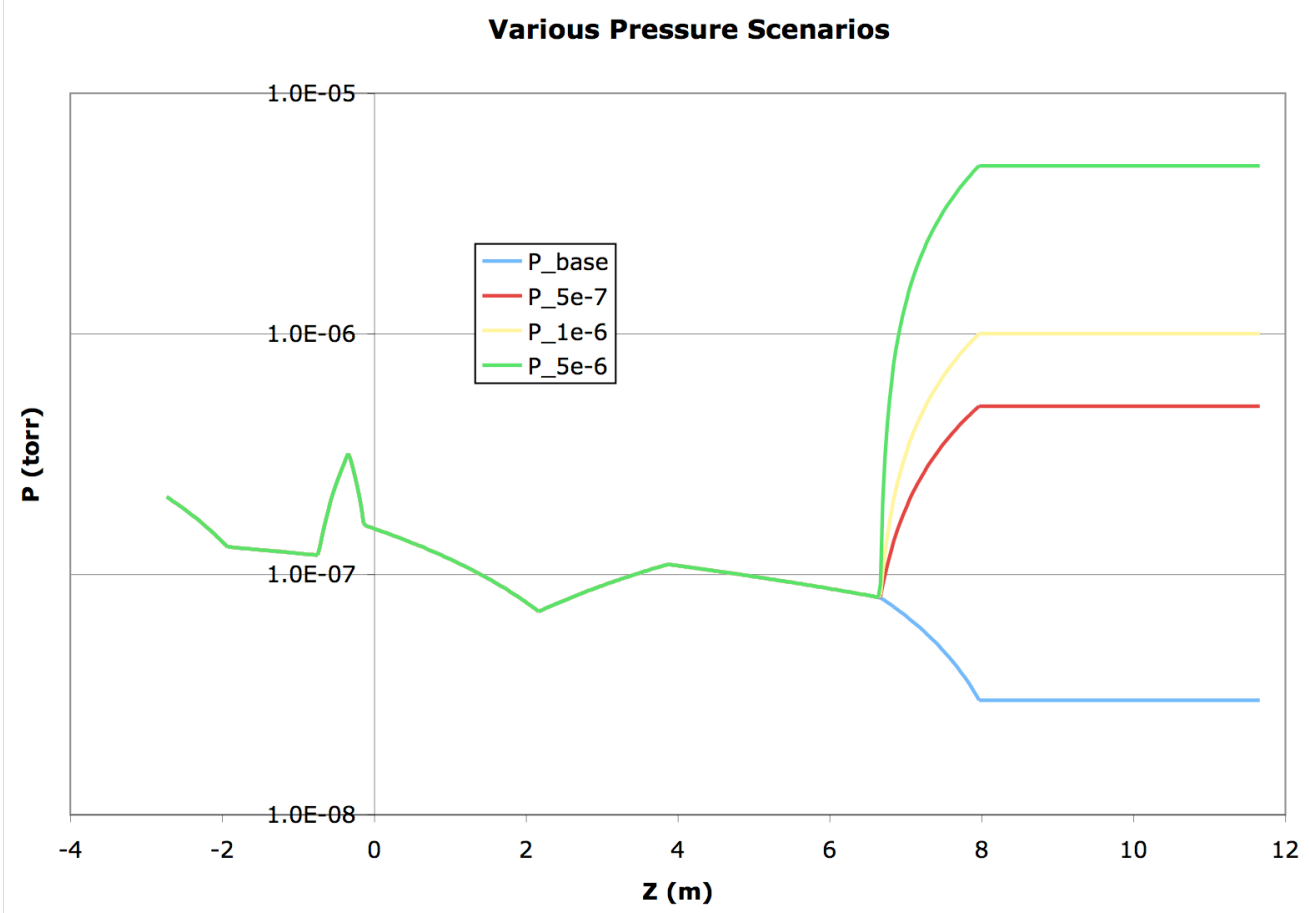

The results of the simulations are shown on the following three pages. In each case, there is a plot of the normalized power spectrum at the end of the transport line, the centroid trajectory at the start and end of the transport line, the beam envelope versus $\mathrm{z}$ at the beginning and end of the pulse, and the beam emittance versus $\mathrm{z}$ at the beginning and end of the pulse. 

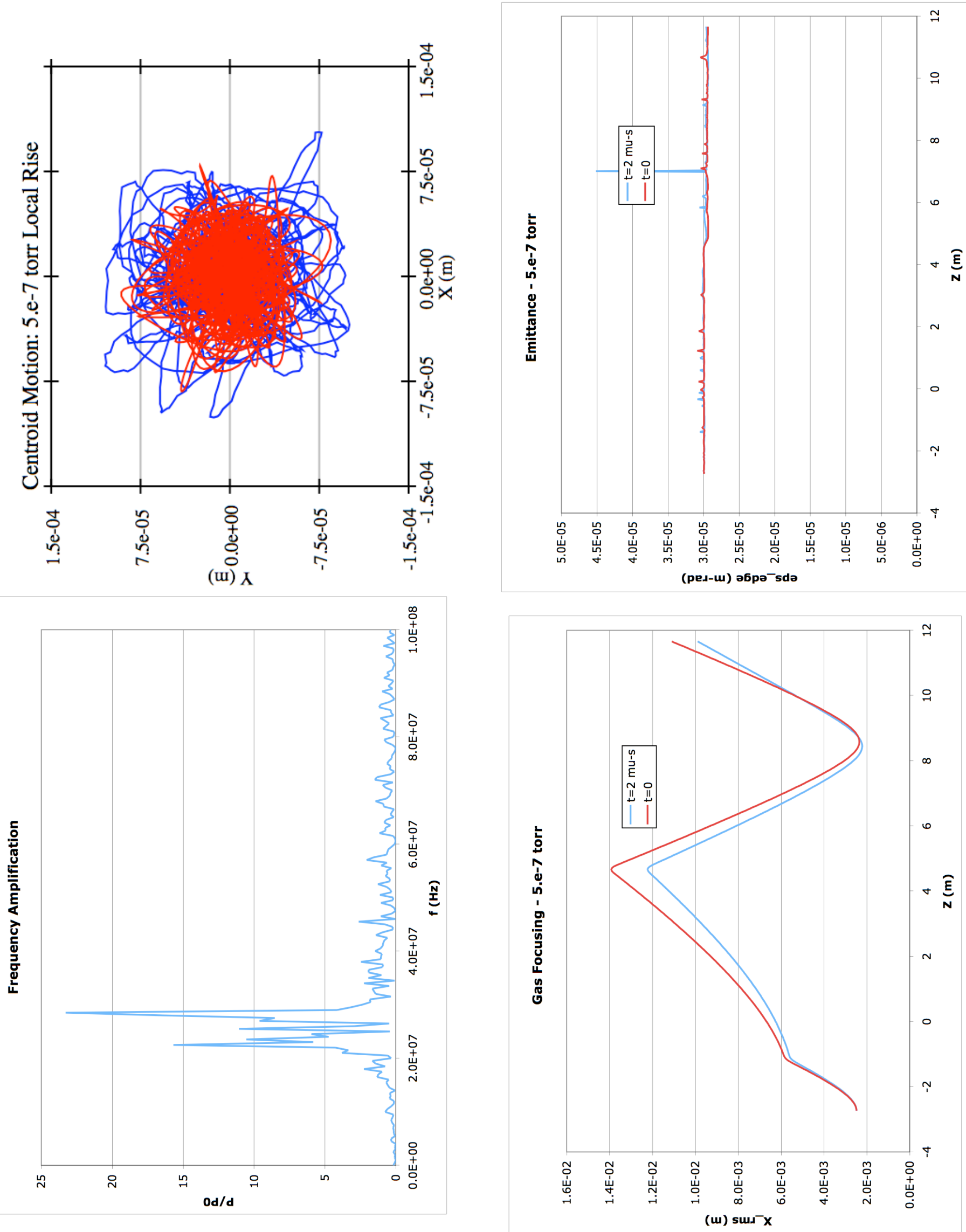

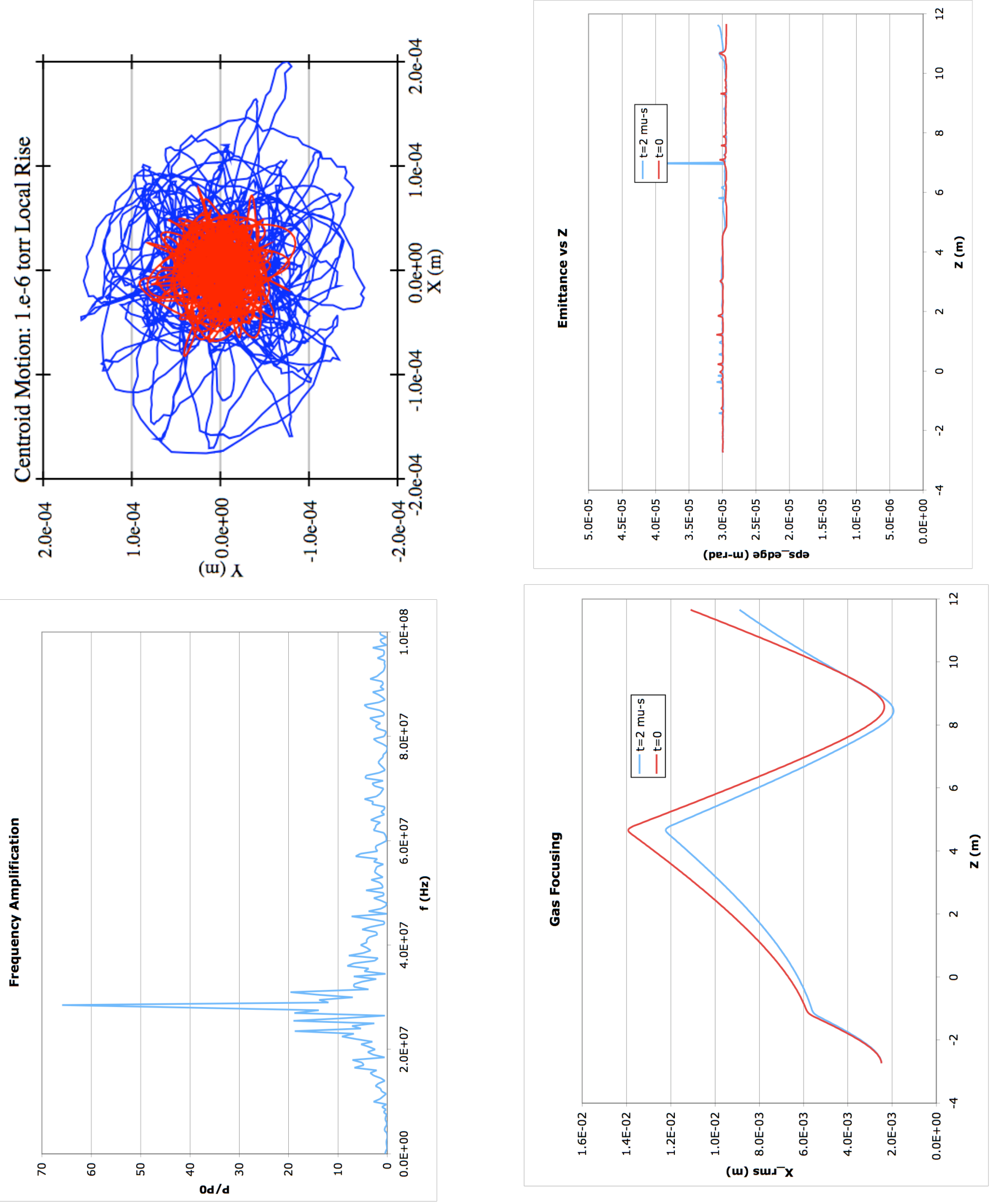

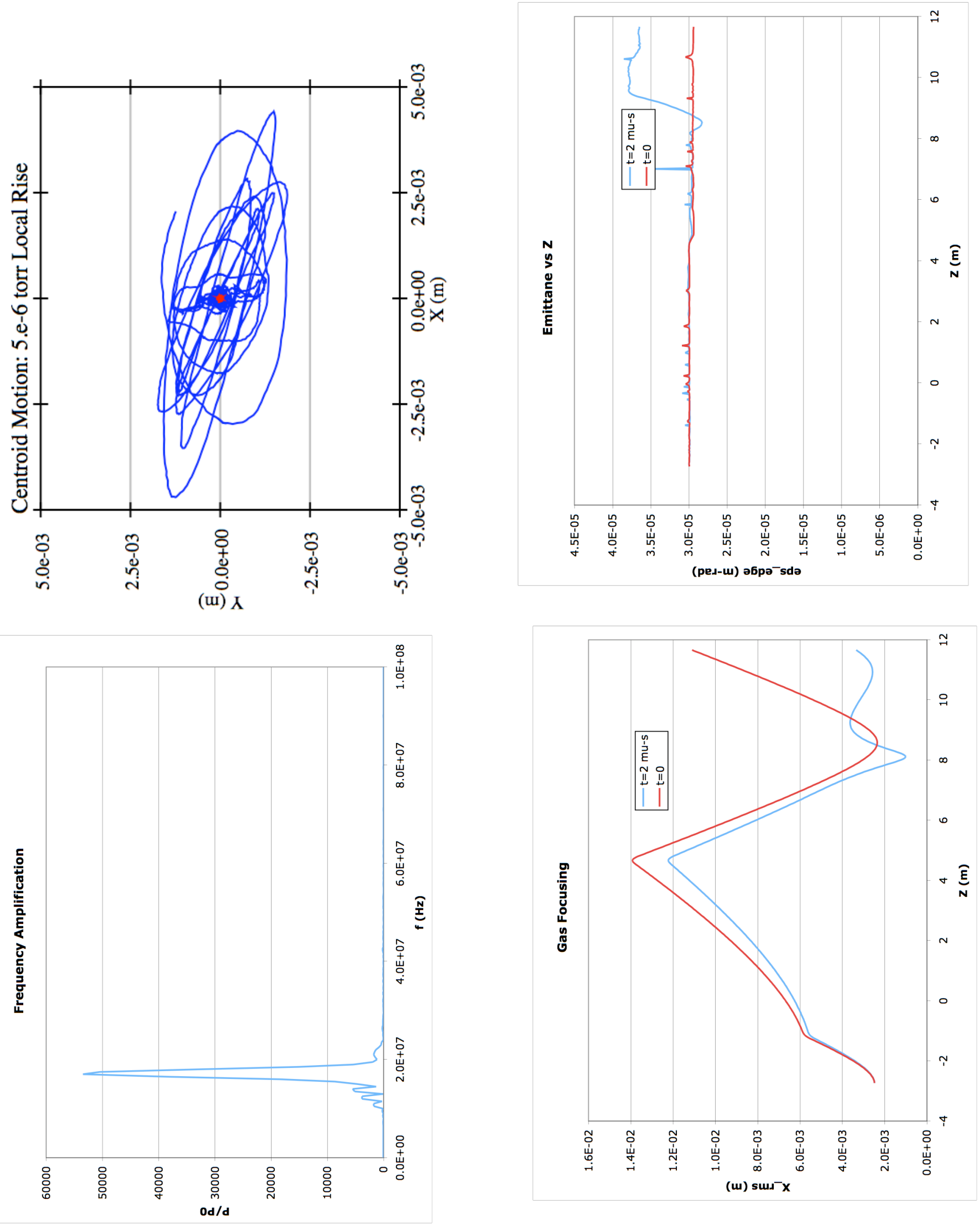
The threshold pressure rise for a localized occurrence is somewhere over $1 \times 10^{-6}$ torr. Note that it is not until the $5 \times 10^{-6}$ torr case that there is emittance growth, and even there it is modest given the large amplitude of the beam motion. Thus it appears safe to say that emittance growth will not be a concern for any acceptable level of centroid motion.

The threshold for strong gas focusing also appears to be at the $1 \times 10^{-6}$ torr level. Note once again that this is defined in terms of a threat to the main dump, not to the quality of transport in the short pulse line.

In perspective, $1 \times 10^{-6}$ torr would be a considerable rise over the baseline. Under this scenario, there is plenty of margin in the safety of the downstream transport with respect to ion hose.

\subsection{Uniform Pressure Profiles}

The baseline pressure profile given in section 8.1 is a calculation, not a measurement. It would be useful to know at what uniform pressure level for the entire $14 \mathrm{~m}$ length does ion hose become problematic; certainly an unexpectedly high pressure along the whole line is a worstcase scenario. Thus here are the results from three additional simulations where the pressure profile is constant in $\mathrm{z}$, at values of $3 \times 10^{-7}, 6 \times 10^{-7}$, and $1 \times 10^{-6}$ torr. Only the centroid trajectories and power spectra are shown, on the following page.

For this scenario, the threshold pressure is lower, in the vicinity of the $6 \times 10^{-7}$ torr case. Note that this is still more than six times higher than the average baseline pressure is expected to be. 

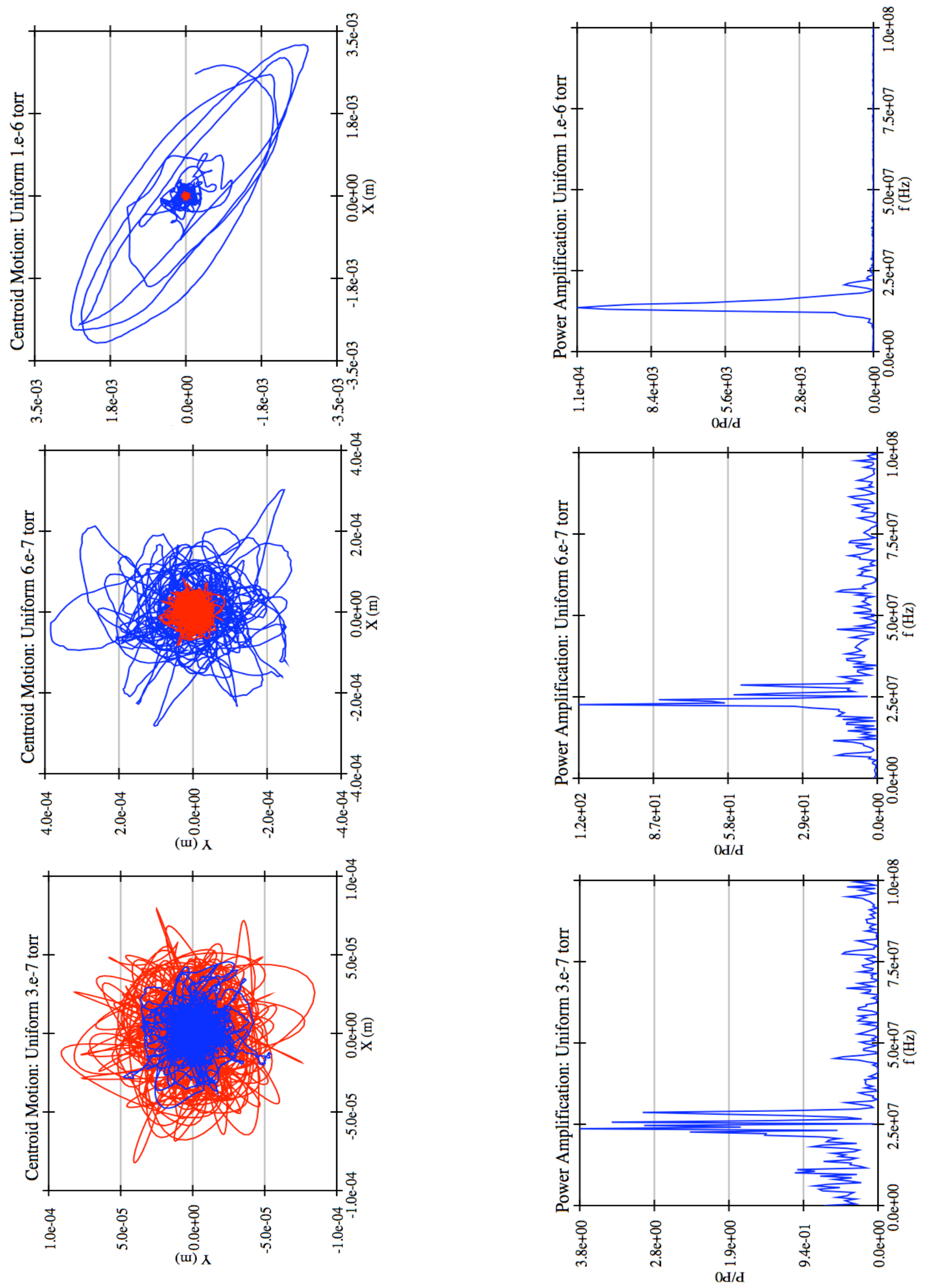


\subsection{Studies with a Gaussian Phase Space}

A valid criticism of the above results is that they are all performed with a $\mathrm{KV}$ phase space for the incoming electron beam. The LSP simulations used as benchmarks used a Gaussian beam, and while the real DARHT-II beam will be neither Gaussian nor KV, it is likely to be much closer to the former. The main concern that has been expressed is that a KV beam has a truly linear radial force on the ions within the body of the beam and so lacks some of the phase-mixing effects present in a more realistic potential. That this should result, if there was a difference at all, in more conservative predictions did not satisfy critics, and so additional runs of the downstream transport have been done with a Gaussian beam to compare with the KV results.

On the subsequent two pages are runs with the baseline pressure profile and a $1 \times 10^{-6}$ torr localized pressure rise. The centroid motion, emittance, and envelopes are largely indistinguishable from their KV counterparts. The power spectrum is somewhat different - the peak is lower but broader. An interesting comparison is the sum under these curves compared to the KV cases:

$\begin{array}{lll}\text { KV } & \text { Baseline } & \underline{1 \times 10^{-6} \text { local rise }} \\ \text { Gaussian } & 21.1 & 664 \\ & 26.3 & 658\end{array}$

So even though the shape of the curves is different, the average amplification is basically the same. This is perhaps not as surprising a result as it might seem if one considers the field profile of the two distributions "everywhere" rather than just inside the beam (which is ill-defined in the Gaussian case). It has been established that ion hose is characterized by a considerably larger ion displacement than beam displacement, so that even for modest ion hose growth (such as the $10^{-6}$ torr case) there will be ions beyond the edge of the KV beam and hence in a nonlinear field. Since the numerical beam edge is defined by a 3000 macroparticle PIC phase space, then an ion creation rate of 40 macroparticles per time step means the beam edge is statistically likely to be found at a time only $0.4 \%$ into the simulation.

There are two additional plots at the end of this section. The first shows the overlap of the ion population relative to the beam edge at the end of the pulse in the $10^{-6}$ torr case (chosen because it has some growth but is still small amplitude) for the KV beam. The ions extend to a distance some $10 \%$ larger than the beam size. There is no equivalent information for the edgeless Gaussian, but let us assume that the ions are themselves smeared over a Gaussian with $\sigma_{\text {ion }}=1.1 \sigma_{\text {beam }}$. The second plot shows the field profiles of the two beams with the intensity of the color weighted by the ion density occupying that radius. Since the "actual" ion density is not used in either case - such information was not preserved in the simulations - these plots are not rigorous proof of anything, but as an estimate of the relative occupancy of the linear versus nonlinear regions of the field profiles, they offer some explanation for why the difference in distributions is not a major factor in the physics of ion hose. 

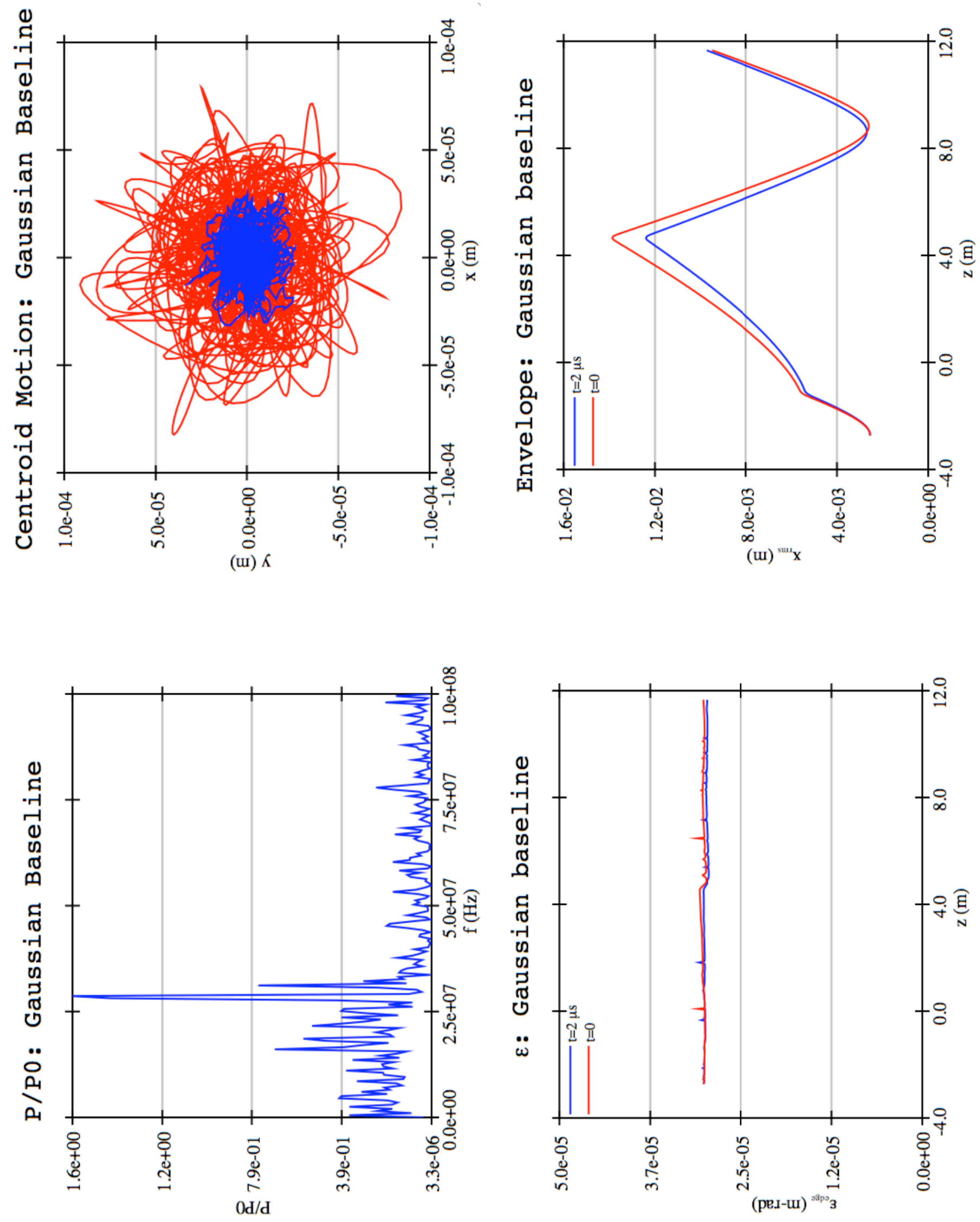

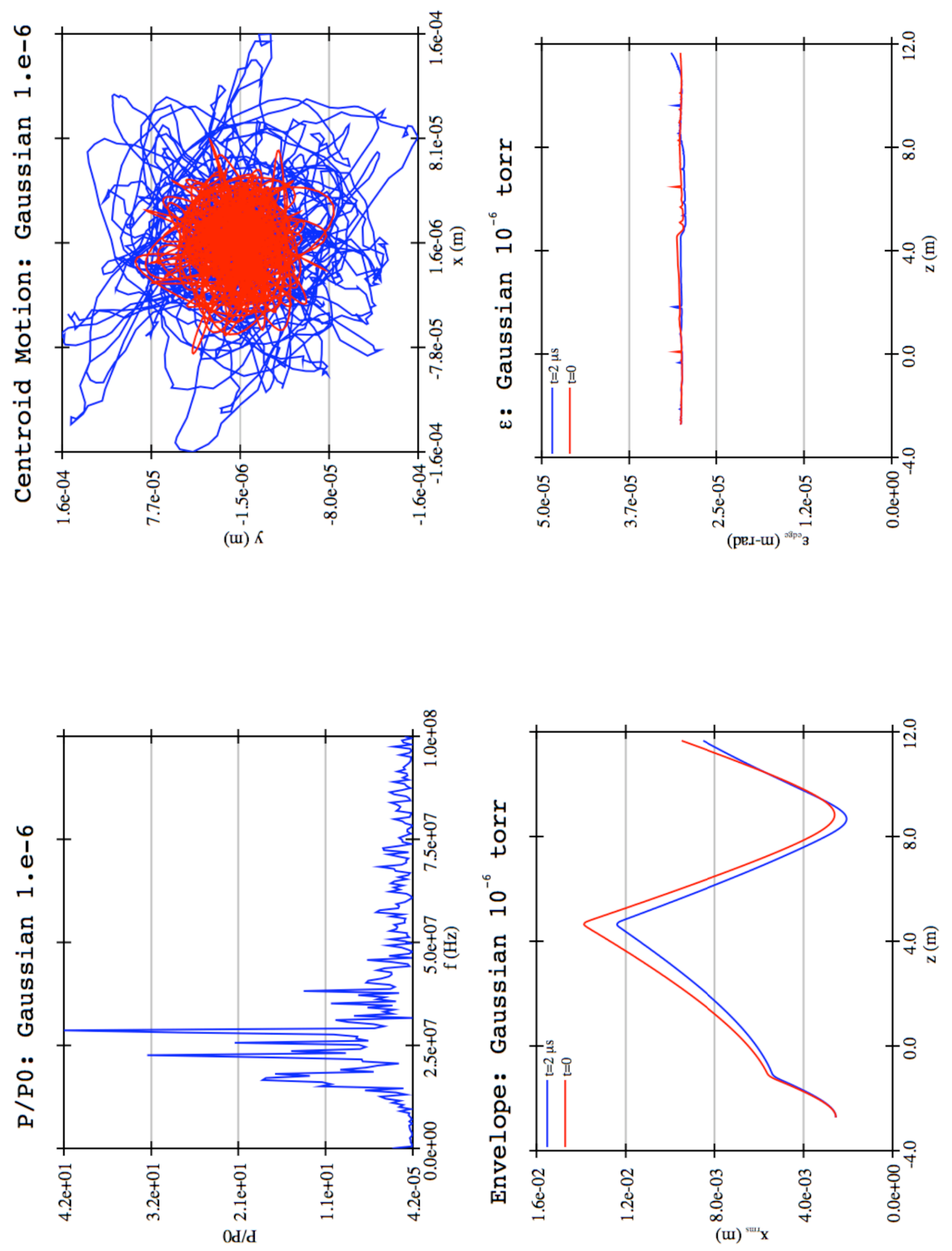


\section{Relative Overlap of $\mathrm{KV}$ Beam and Ions}

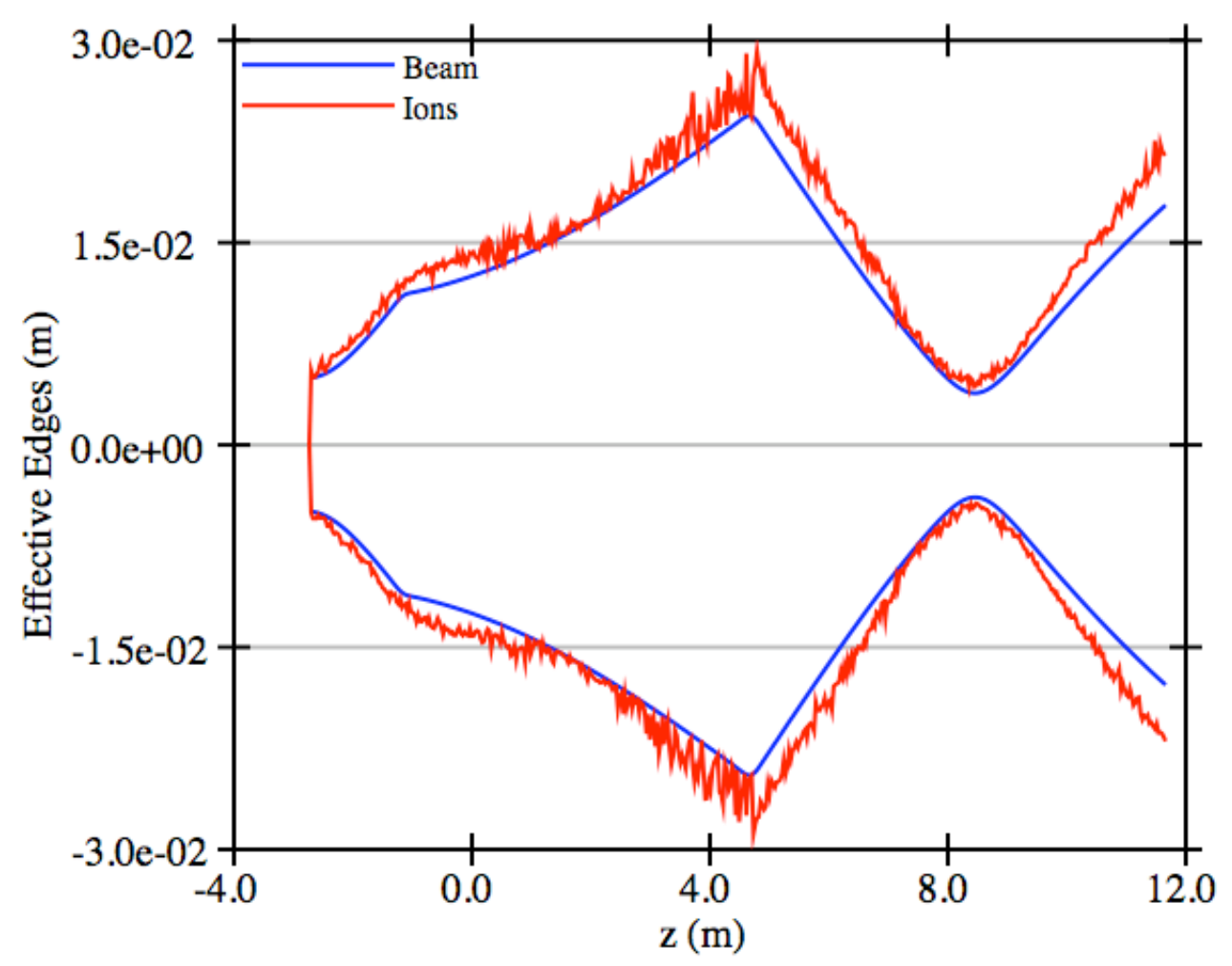

Occupancy-Weighted Field Profiles

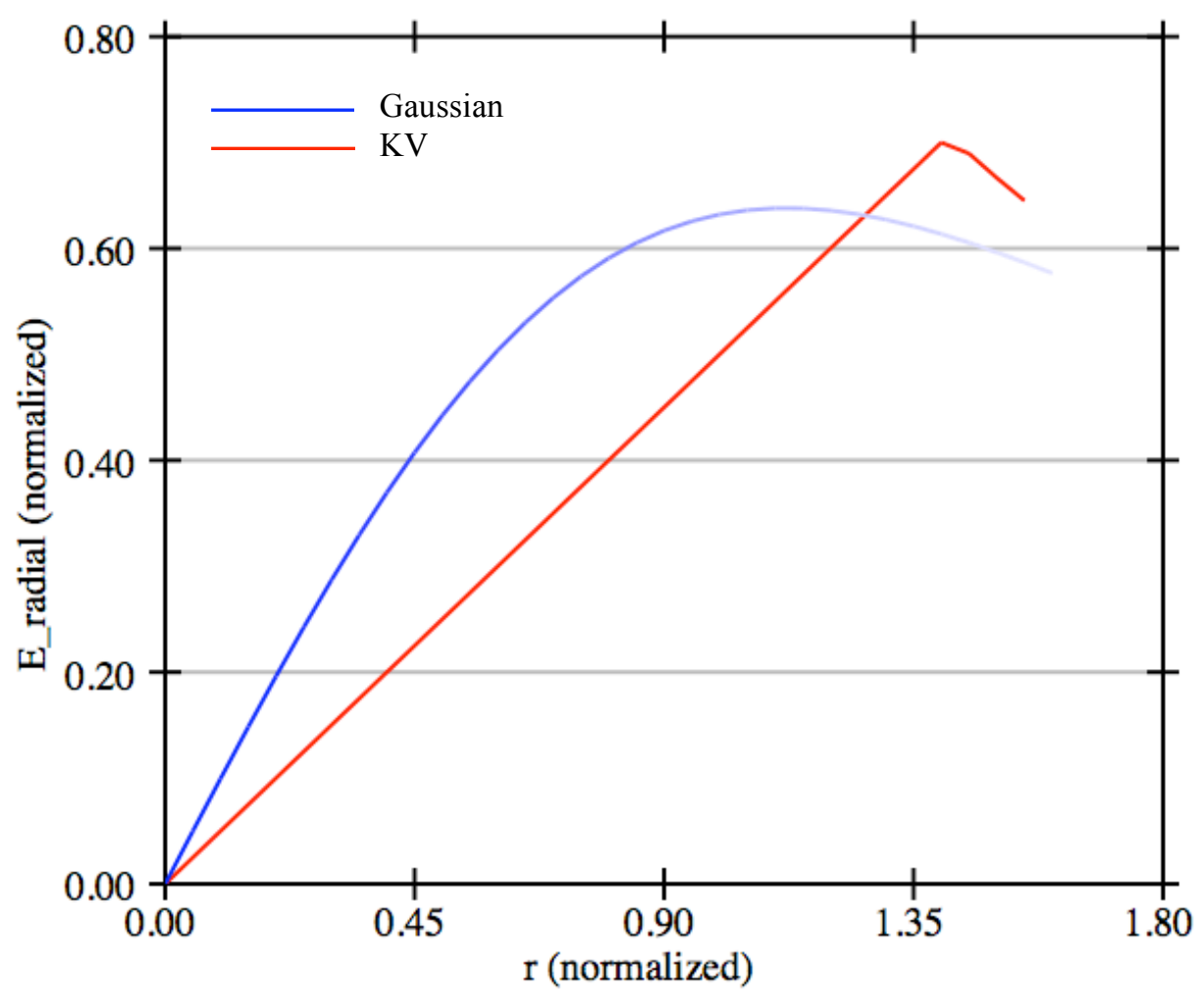




\section{Proposed Experiment to Verify Results}

Ion hose over this scale of time and length is a complex problem and it would be foolhardy to put blind faith in purely computational conclusions. An opportunity exists to perform a simple set of experiments to verify the predictive capabilities of the code. There is a planned series of scaled experiments to look at the beam stability (in the presence of BBU and perhaps ion hose also) within the DARHT-II accelerator, at an energy of $8 \mathrm{MeV}$ and a current of $1.4 \mathrm{kA}$. It would be a simple matter to piggyback some downstream transport experiments onto this configuration as well.

One limitation to this experiment is that the downstream line will terminate only about $5 \mathrm{~m}$ from the accelerator exit, at the location of the "shuttle" dump whose specific purpose is machine commissioning. There are no other transport elements on the line. In order to see convincing evidence of ion hose under these circumstances, it may be necessary to deliberately raise the pressure in that downstream reason to fairly high levels $\left(\sim 1 \times 10^{-6}\right.$ torr $)$.

In addition to the pressure, there is another knob: the size of the beam exiting the accelerator. This can be used to verify the importance of envelope variation in reducing the ion hose growth rate. A small, emittance-dominated beam leaving the accelerator will expand rapidly, whereas a large beam will not.

The data collection and reduction for these experiments is very similar to the types of results that come directly from the simulation. A beam position monitor at the end of the accelerator and just before the shuttle dump can be used to record the centroid motion with time. A power spectrum analysis of that data should provide results directly comparable with those of the simulation.

On the following pages are simulations of these experiments, in a uniform pressure profile with magnitudes of $5 \times 10^{-7}, 8 \times 10^{-7}$, and $2 \times 10^{-6}$ torr. Two beam sizes exiting the accelerator are used, 5 $\mathrm{mm}$ and $2 \mathrm{~cm}(\mathrm{rms})$. The first page shows plots of the beam envelope with background gas focusing, which shows that the pressure and the beam envelope are not truly decoupled effects at these levels. The plots on the second page show that strongly measurable power amplification does not occur until pressure into the $10^{-6}$ range, and then only for the small beam. It is interesting to note that the small initial beam size, with significant subsequent envelope variation, leads to a stronger ion hose effect than for the larger beam with flatter envelope. 


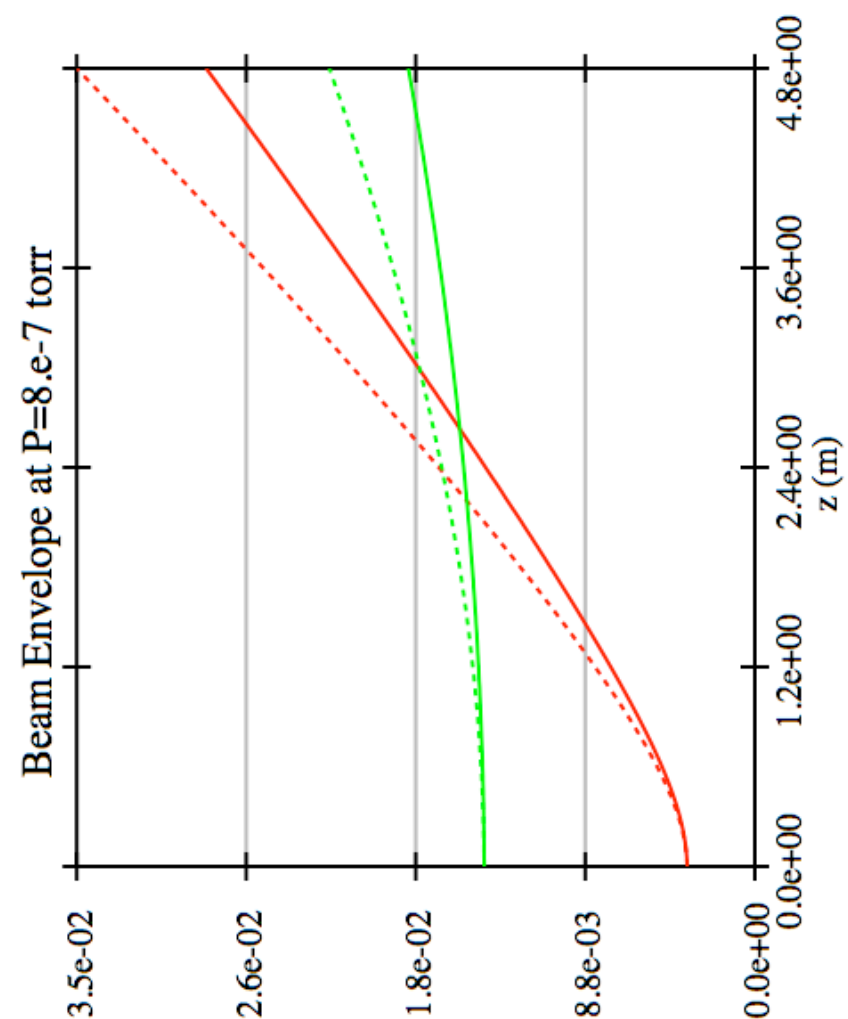

(w) $\operatorname{sun}^{-} \mathrm{x}$

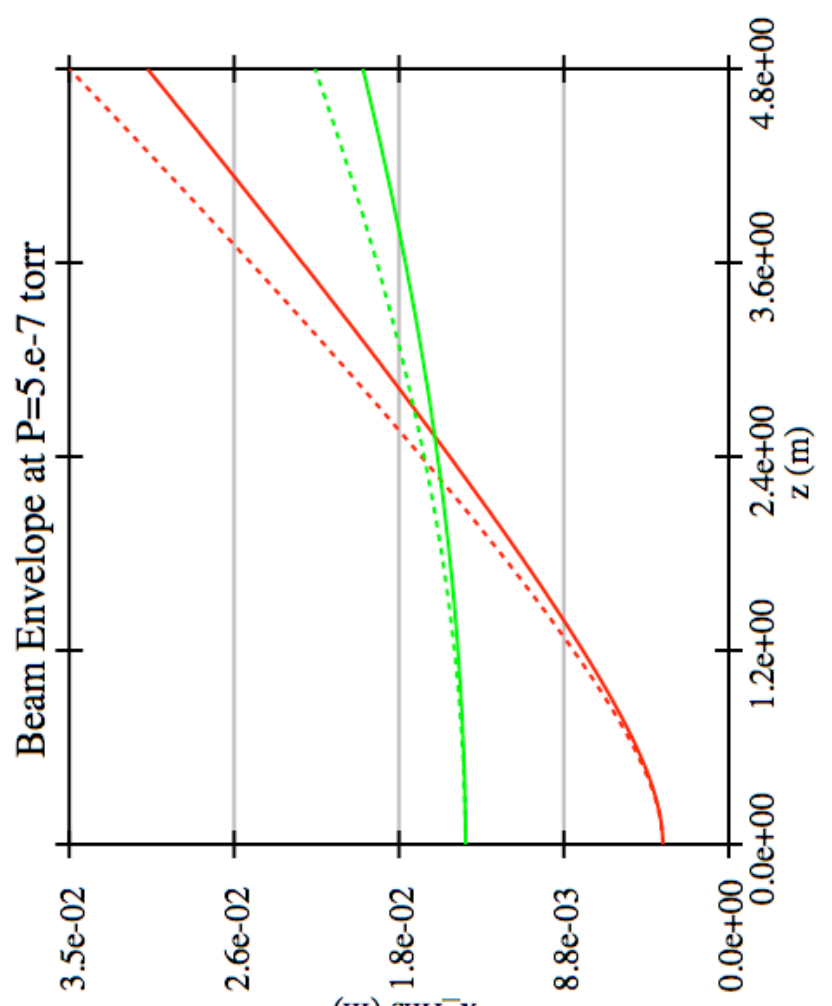

(ui) $\operatorname{suli}^{-} \mathrm{x}$

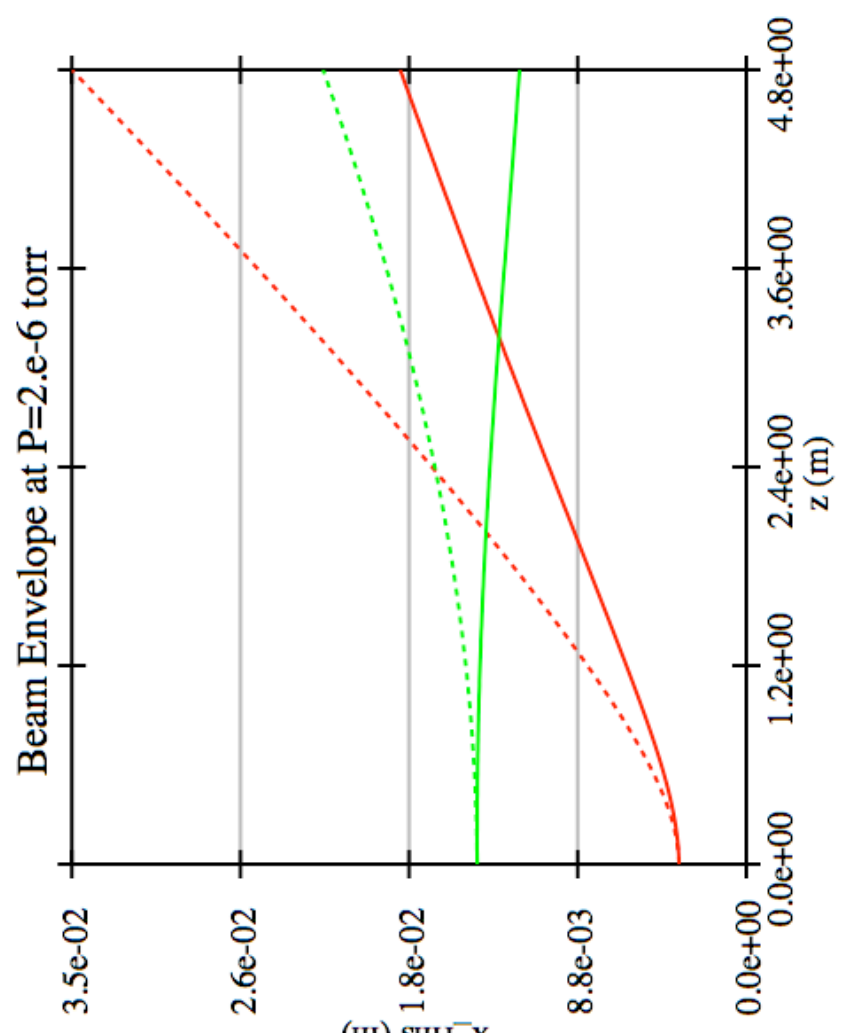

(ui) suli $^{-} \mathrm{X}$

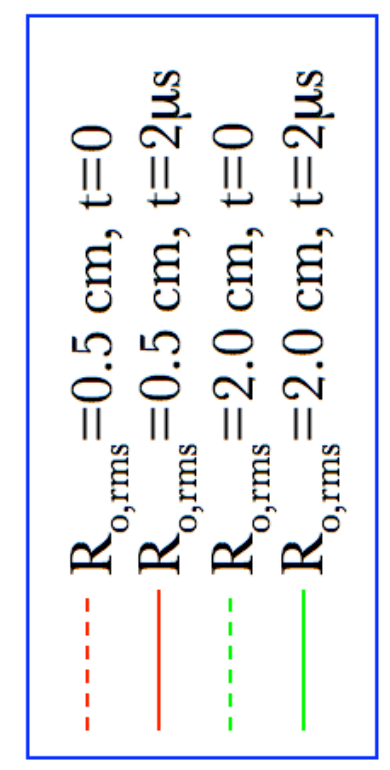



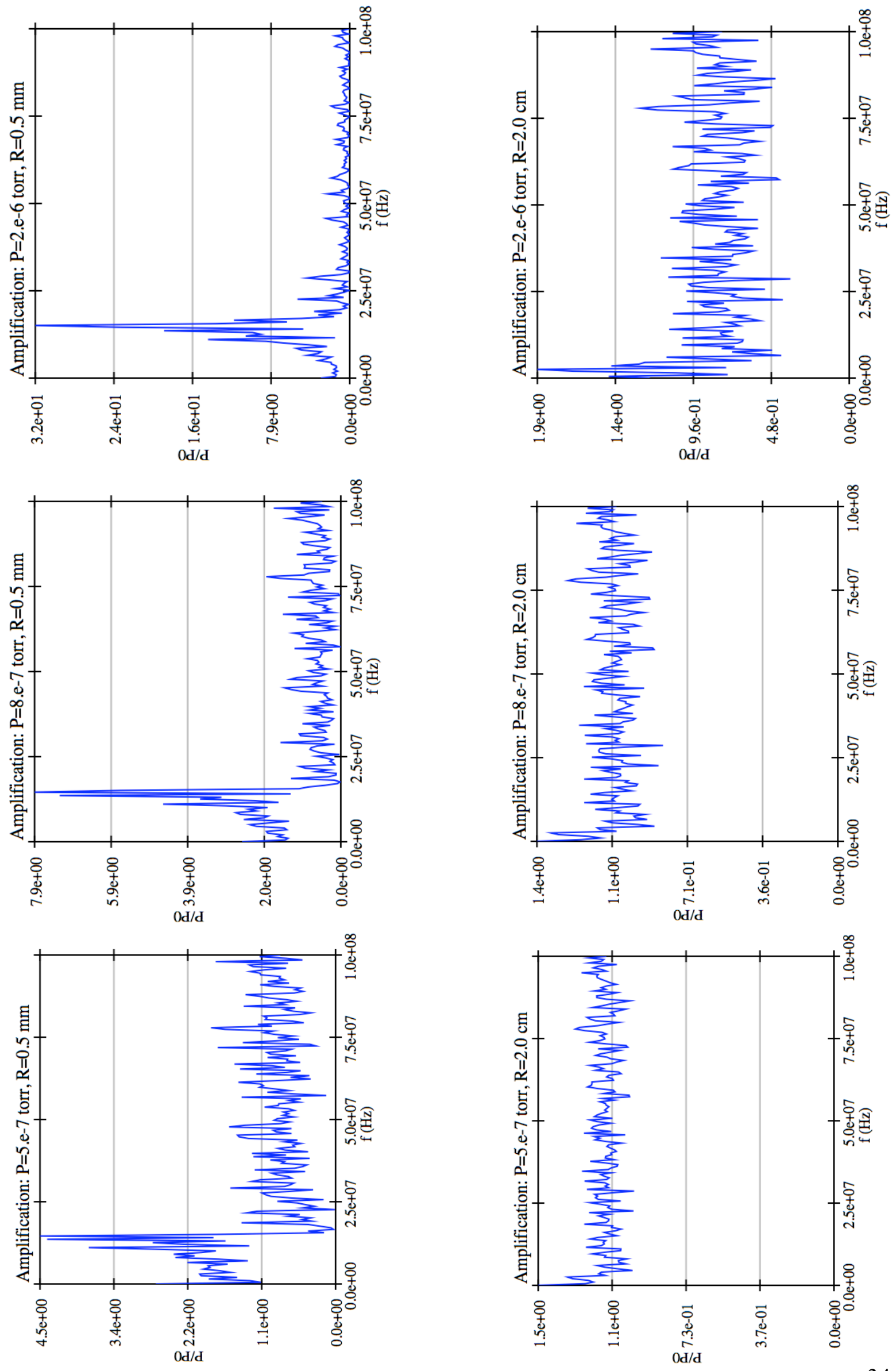


\section{Conclusion}

In this report, we have studied the effects of the ion hose instability and background gas focusing in the downstream transport section of the DARHT-II accelerator and found that they are not significant, with appreciable margin of safety.

The ADCGlib beam physics library was used to perform a series of simulations. First, a set of benchmark calculations was performed to compare ADCGlib results with those published in the literature. The comparison has some outstanding issues but is good enough for the purpose at hand. Then, a number of scenarios in the downstream transport line were considered. The baseline case, using a predicted background pressure profile, shows no effects of ion hose, gas focusing, or emittance growth. Localized pressure increases relative to this baseline, meant to simulate the effect of gas desorption from the septum region and main dump due to beam heating, do not have an effect until extreme levels, more than thirty times the baseline value. A uniform pressure profile over the $14 \mathrm{~m}$ transport length would not have an effect until a value six times higher than the average of the baseline.

In all scenarios, centroid motion and gas focusing become significant at about the same pressure level. Emittance growth is not appreciable until after the other effects become serious. A preliminary comparison of runs done with a Gaussian beam to the results with a KV beam shows that the details of the beam distribution are not important.

A simple experiment to verify these simulations is proposed as an add-on to an existing set of DARHT-II experiments planned for $8 \mathrm{MeV}, 1.4 \mathrm{kA}$ operation. The experiments should provide results that are easily compared with code, namely the power spectrum amplification of the centroid motion over a $5 \mathrm{~m}$ drift space, at high pressure.

\section{References}

1. Burns M.J., et al. "Status of the Dual Axis Radiographic Hydrodynamics Test (DARHT) Facility." BEAMS 2002, Albuquerque, NM, pp. 139-142.

2. Chen Y.J. et al. "Experimental Results of the Active Deflection of a Beam from a Kicker System.” LINAC 1998, Chicago, Il, pp. 1007-1009.

3. Genoni T.C., Hughes T.P, "Ion Hose Instability in a Long Pulse Linear Induction Accelerator." PRST-AB vol. 6, 2003.

4. Behne D., Bertolini L. "Vacuum Desorption Studies of DARHT II Beam Dump Materials." UCRL-ID-147135, Section 4.2. This document is export controlled.

5. Hughes T.P., Davis H. "Effect of Stimulated and Thermal Desorption in DARHT-2." PAC 2003, Portland, OR, pp. 120-122.

6. Bers A. "Space-Time Evolution of Plasma Instabilities - Absolute and Convective." Handbook of Plasma Physics, Vol. 1, North-Holland Publishing 1983, pp. 451-517. 
7. Buchanan H.L. "Electron Beam Propagation in the Ion Focused Regime." Physics of Fluids vol. 30, no. 1, Jan 1987, pp. 221-231.

8. Chen Y-J., et al. "Downstream Transport System for the Second Axis of the Dual-Axis Radiographic Hydrotest Facility." BEAMS 2002, Albuquerque, NM, pp.147-150.

9. Hughes, T.P., private communication.

10. Bertolini L., et al. "DARHT II Downstream Transport Vacuum System Calculations." UCRL-ID-147135, Section 4. This document is export controlled.

11. Paul, A.C. "The DARHT2 External Beamline." UCRL-ID-147135, Section 1. This document is export controlled.

\section{Appendix: The MPI Version of the Main Algorithm}

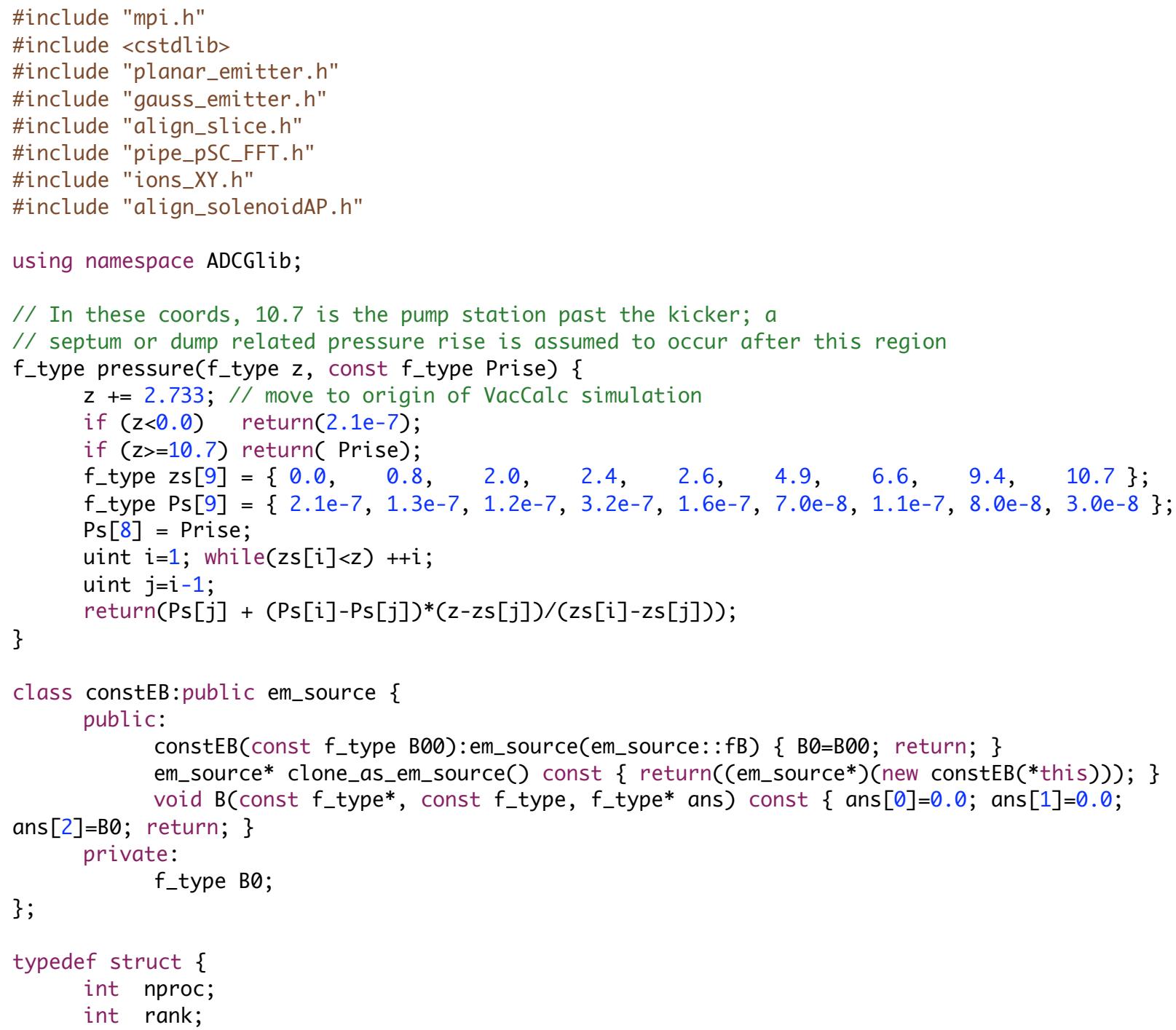




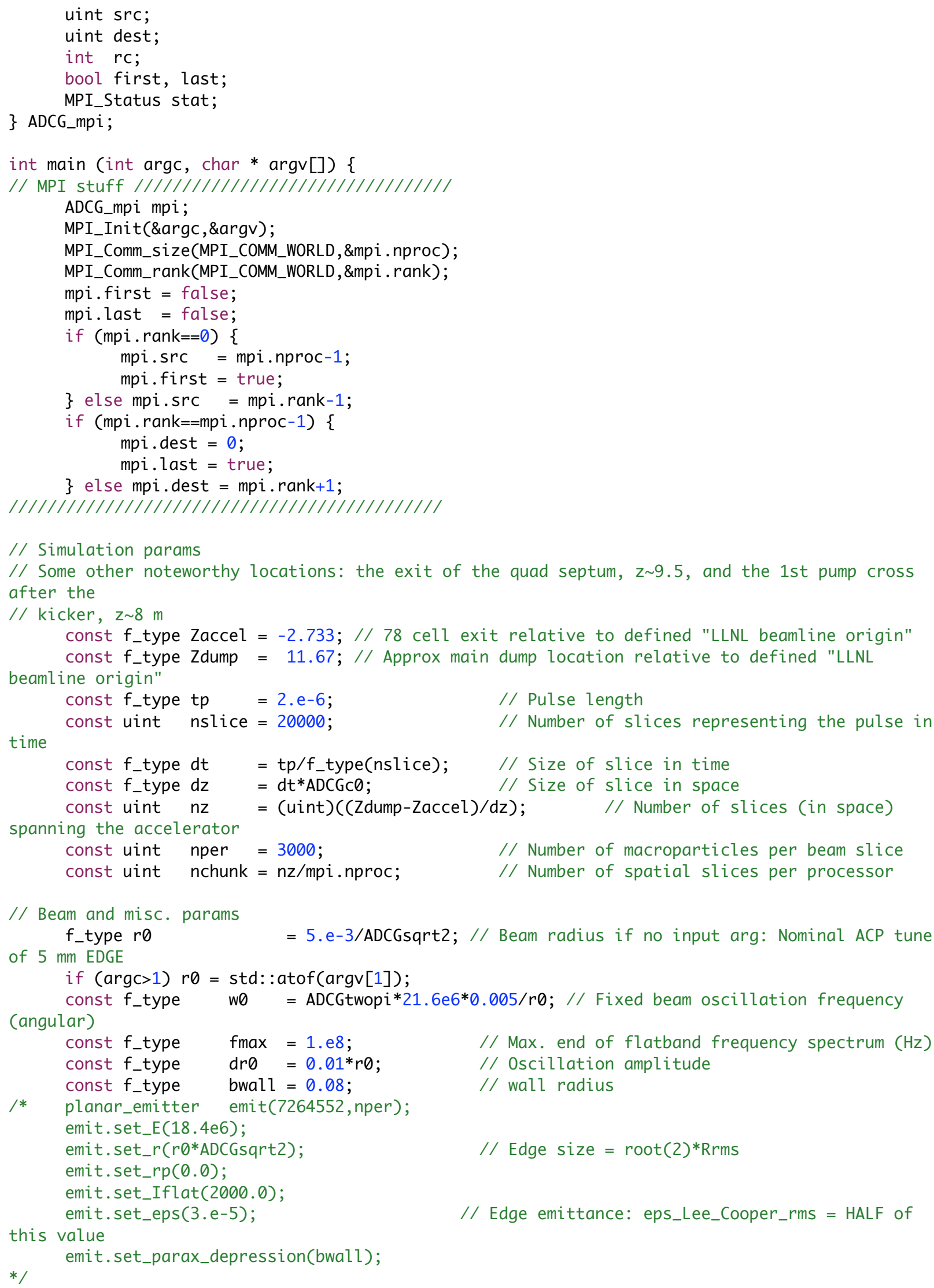




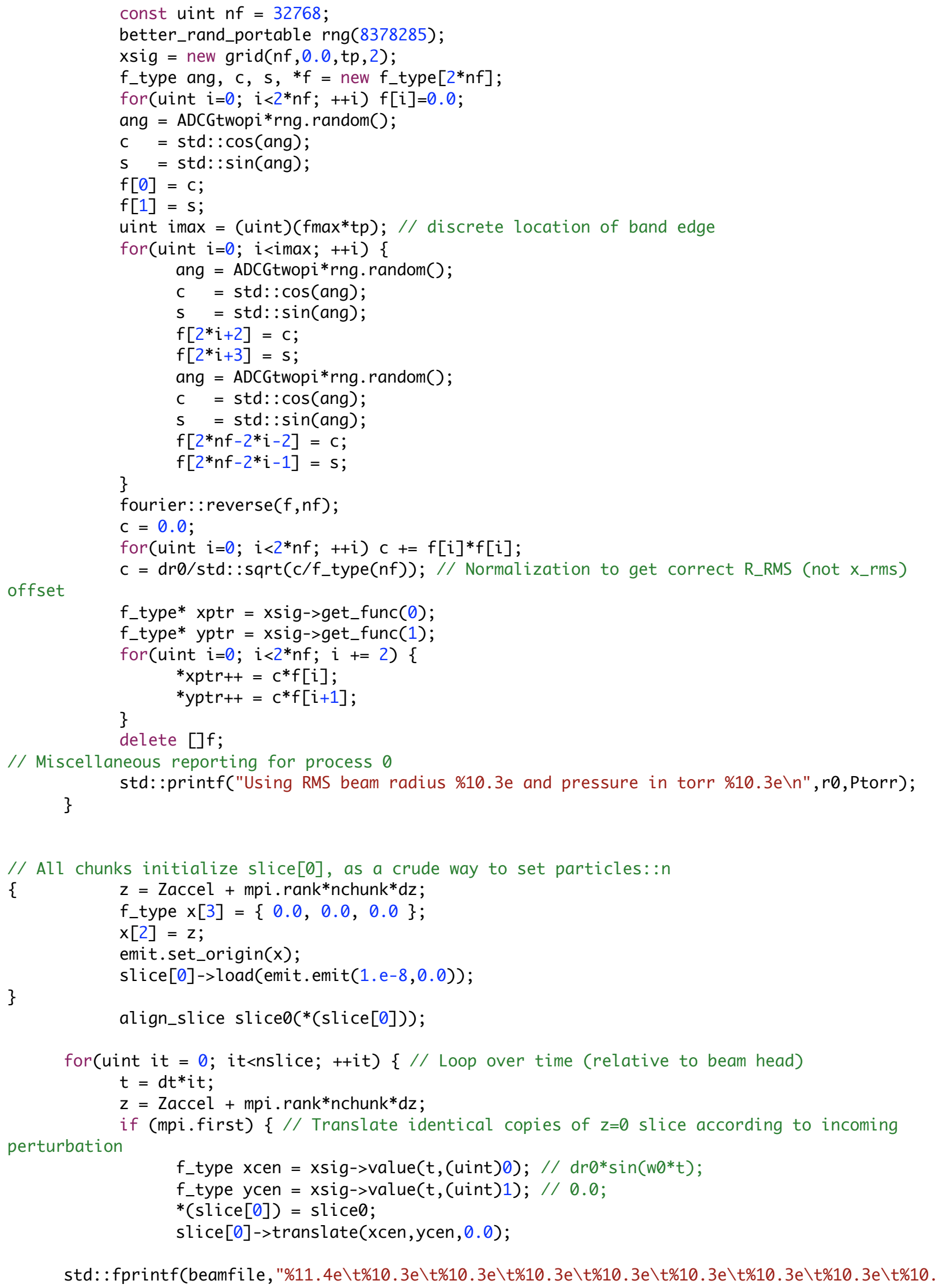




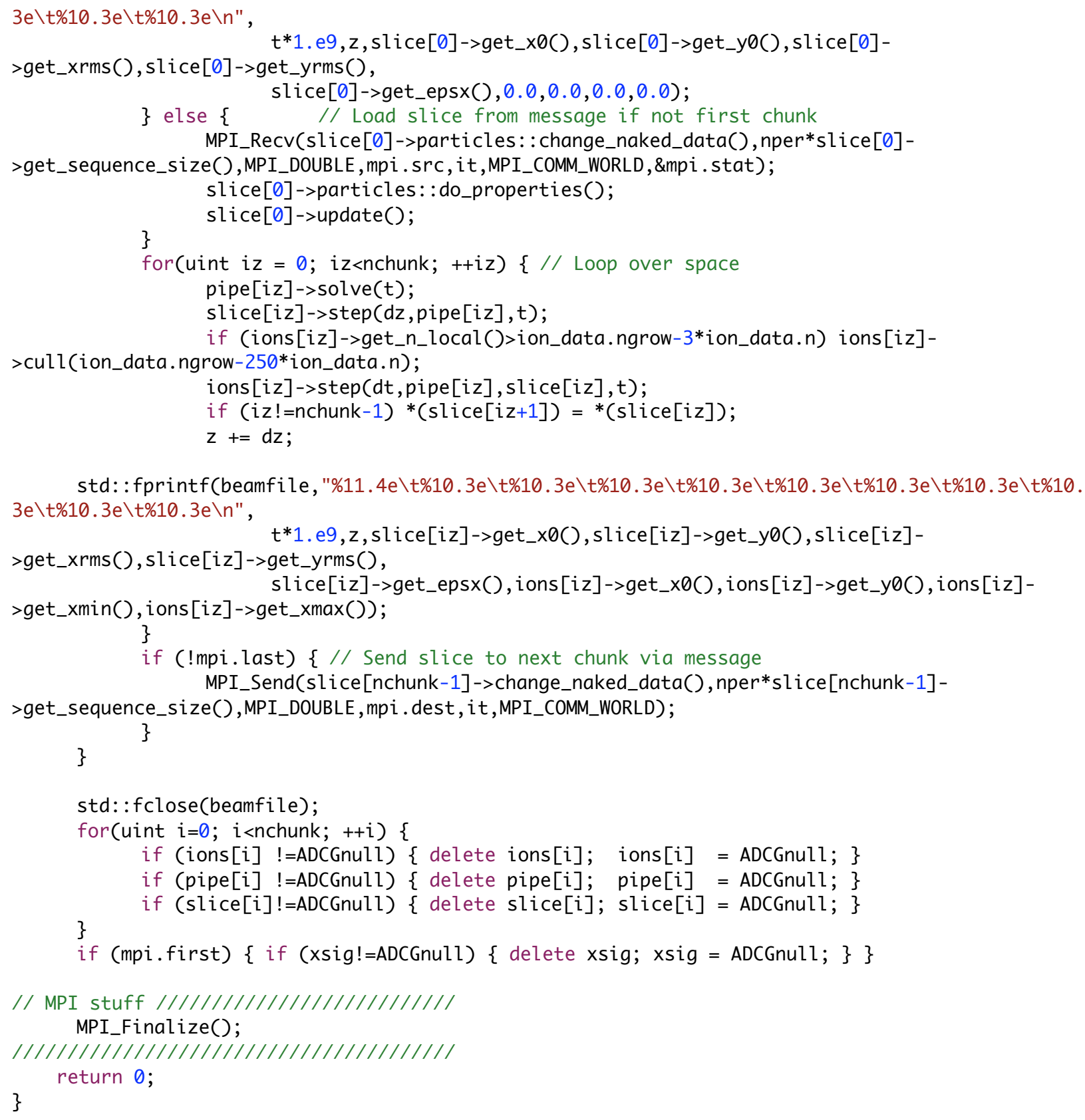

